\title{
Electronic Polarization Reversal and Excited State Intramolecular Charge Transfer in Donor/Acceptor Ethynyl Pyrenes
}

\author{
Shu-Wen Yang, ${ }^{\dagger}$ Arumugasamy Elangovan, ${ }^{\dagger}$ Kou-Chu Hwang ${ }^{\ddagger}$ and Tong-Ing Ho* ${ }^{\dagger}$ \\ ${ }^{\dagger}$ Department of Chemistry, National Taiwan University, Taipei - 106, Taiwan, ${ }^{\dagger}$ Department of \\ Chemistry, National Tsing Hua University, Hsin Chu-300, Taiwan
}

Fax: +886-2-23636359; E-mail: hall@ntu.edu.tw

Supporting Information

Contents

Page \#

1. Proton NMR spectra of Pyrene

S2-S6

2. ${ }^{13} \mathrm{C}$ NMR spectra of Pyrene...

S7-S10

3. CV curves of Pyrene.

$\mathrm{S} 11-\mathrm{S} 13$

4. Raw ECL spectra

S14-S15

5. Solvatochromic data tables

S16-S17

6. Plot of polarizability ( $\Delta \mathrm{f}$ and $\pi^{*}$ ) of the solvent

S18-S19

7. Concentration quenching of fluorescence spectra.

$\mathrm{S} 20-\mathrm{S} 21$

8. Cartesian coordinates of all molecules

$\mathrm{S} 22-\mathrm{S} 28$

9. Fluorescence lifetime data for all compounds

S29-S31 


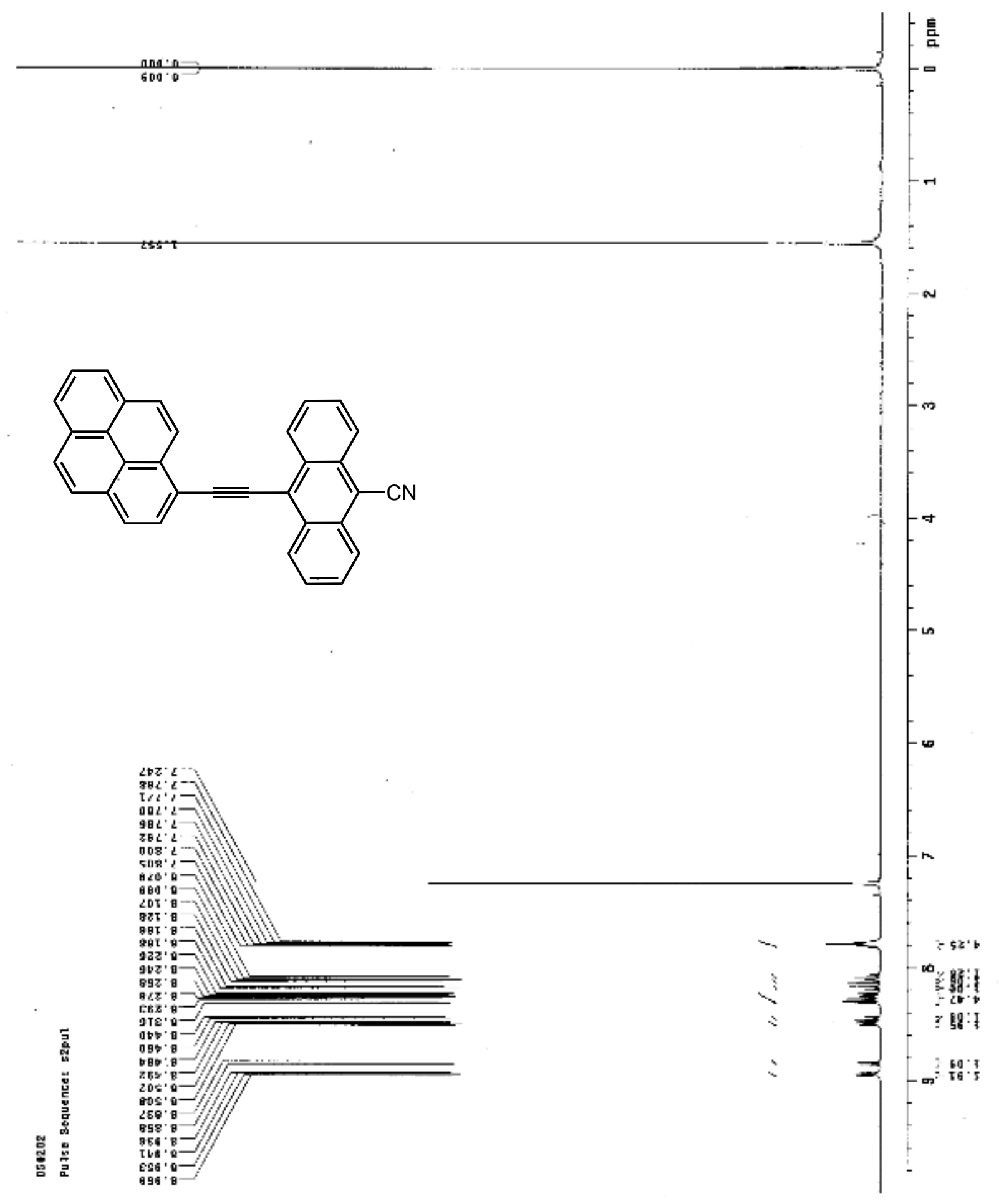




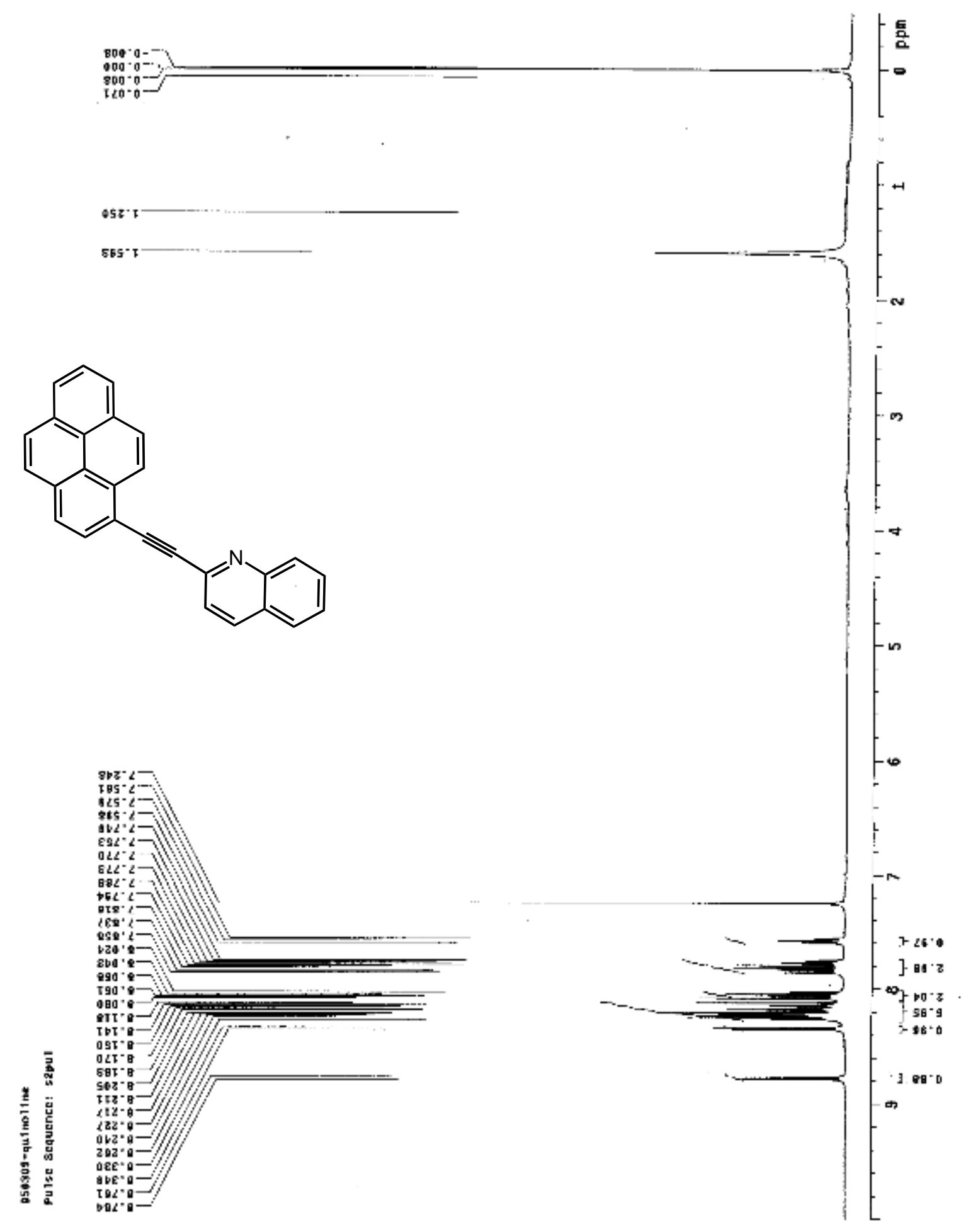



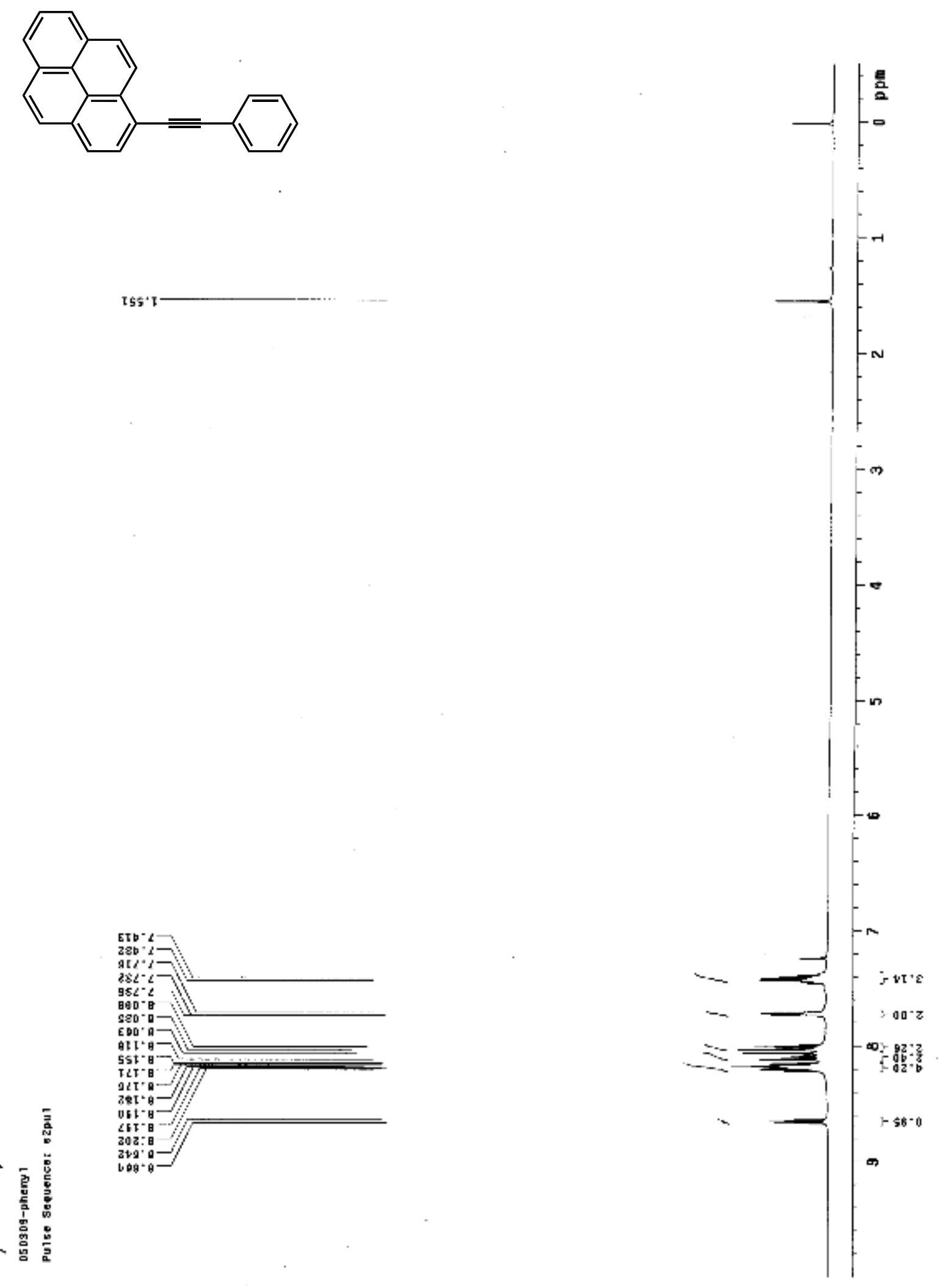


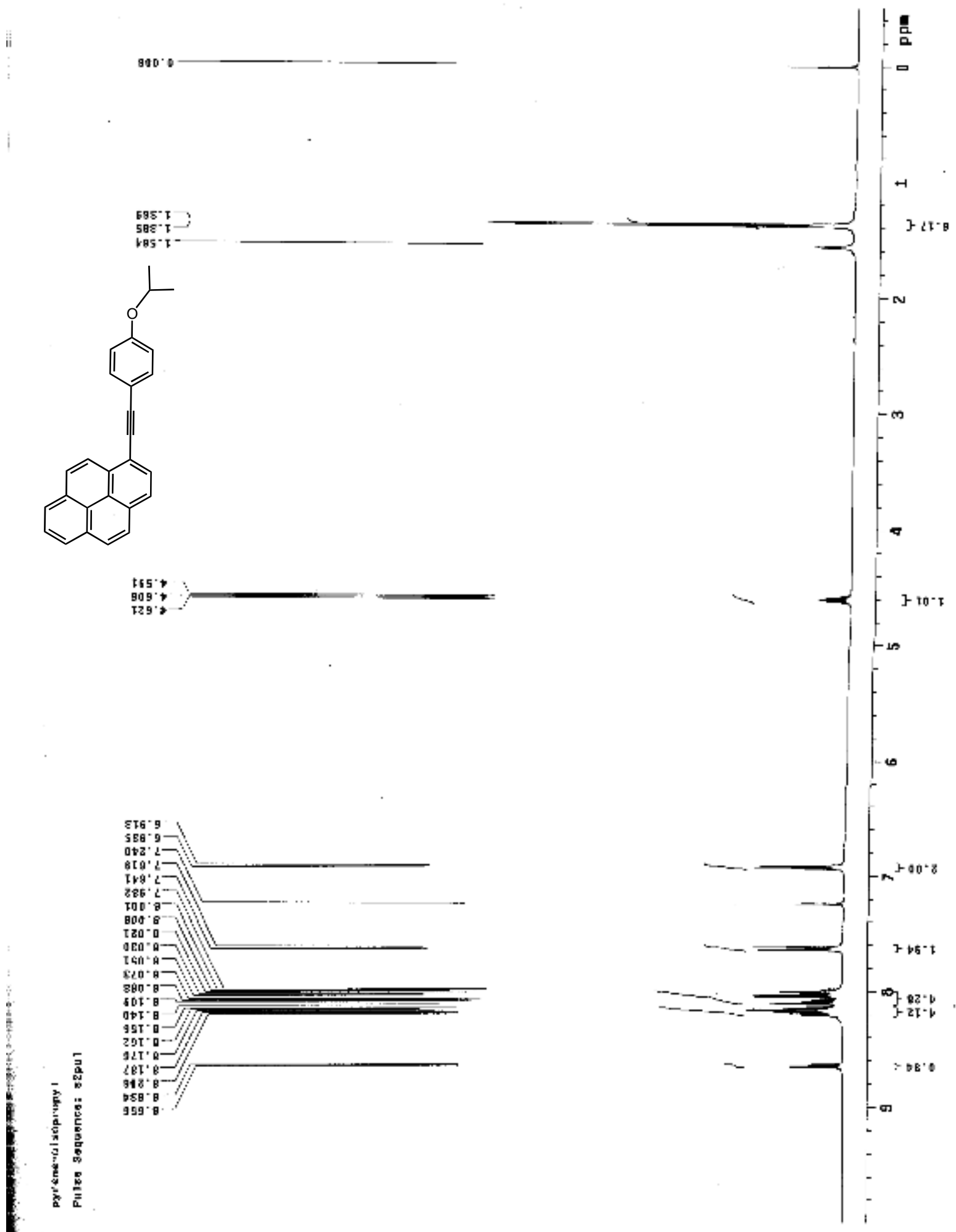




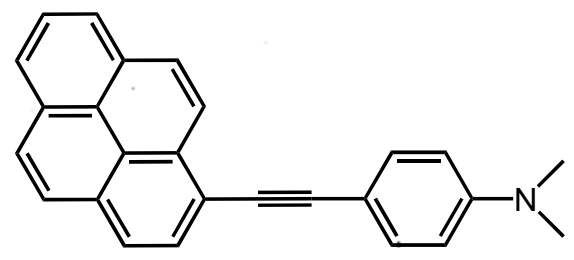

234'E-
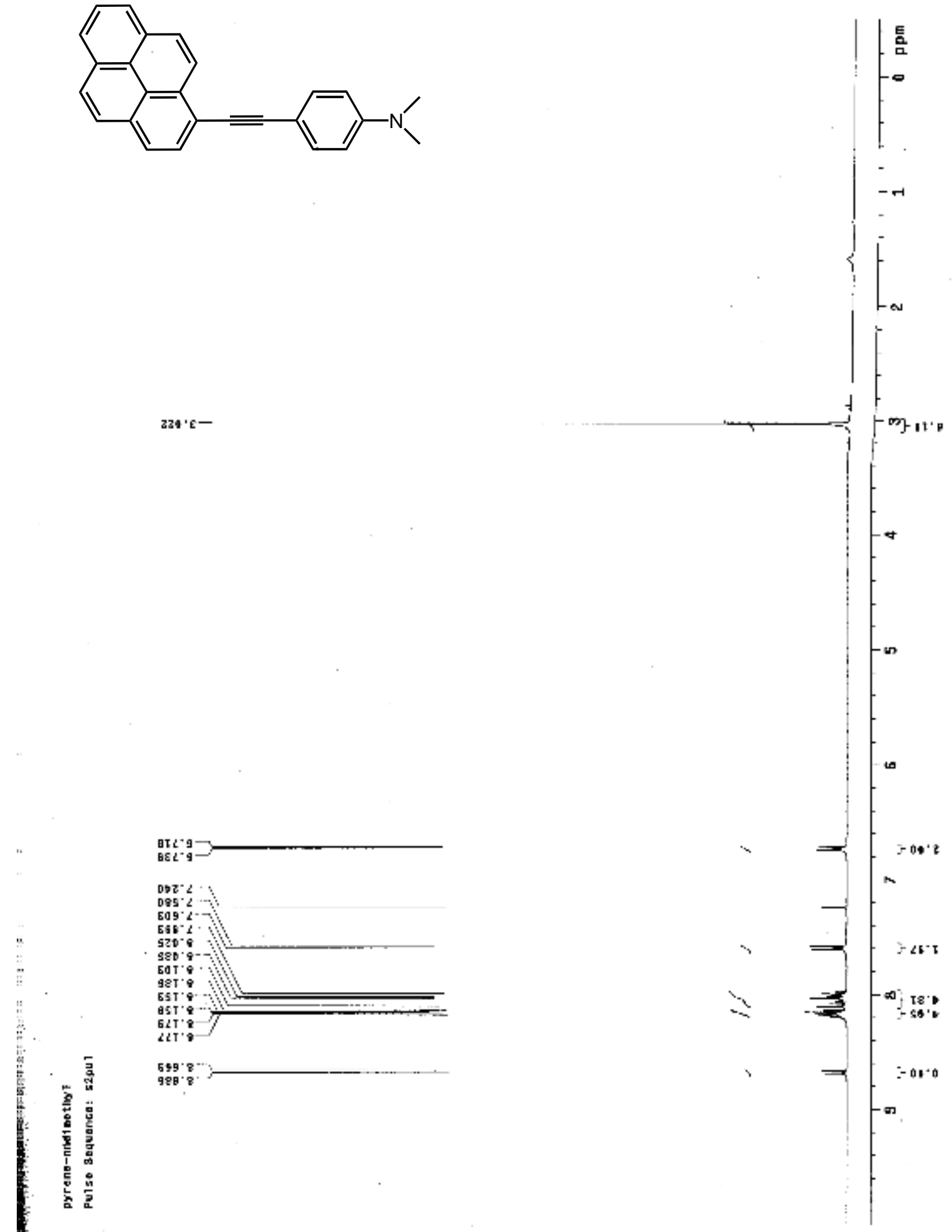

S6 
9.89' 12

$000 \cdot 22=$

arz' 18

นดL 3

890.

geเ 82

BOT'bZT

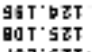

$49 \mathrm{~g} \cdot 5 \mathrm{STT}$

Th8

099 ' 925

ETL' $92 \mathrm{~T}$

98L' 927

LT' $12 \mathrm{~T}$

COZ'

3 I ' 221

COQ'目列

at S'0eT

โร้' โริ

हล' $2 \mathrm{~T}$

ESO'GET

TA2'EDT
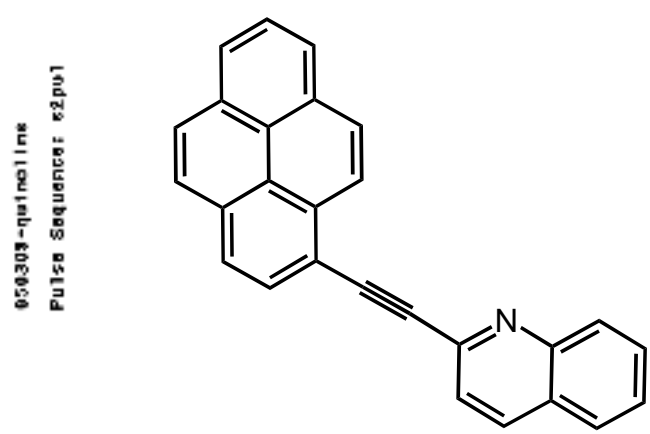

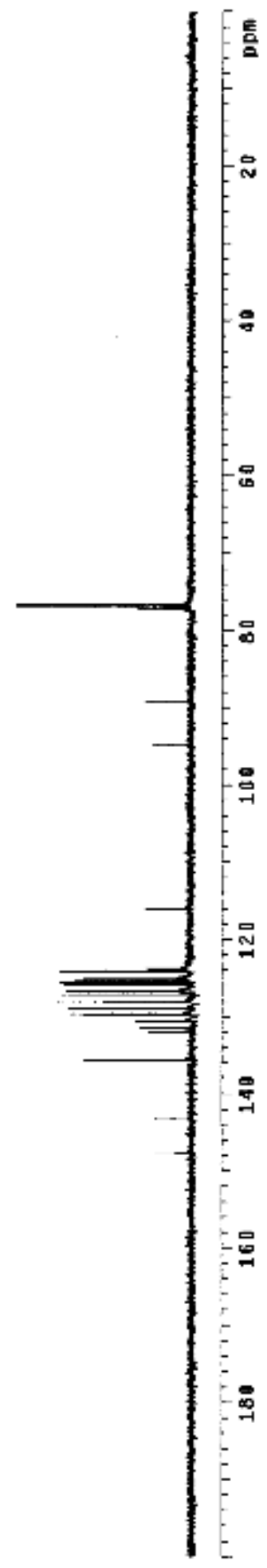




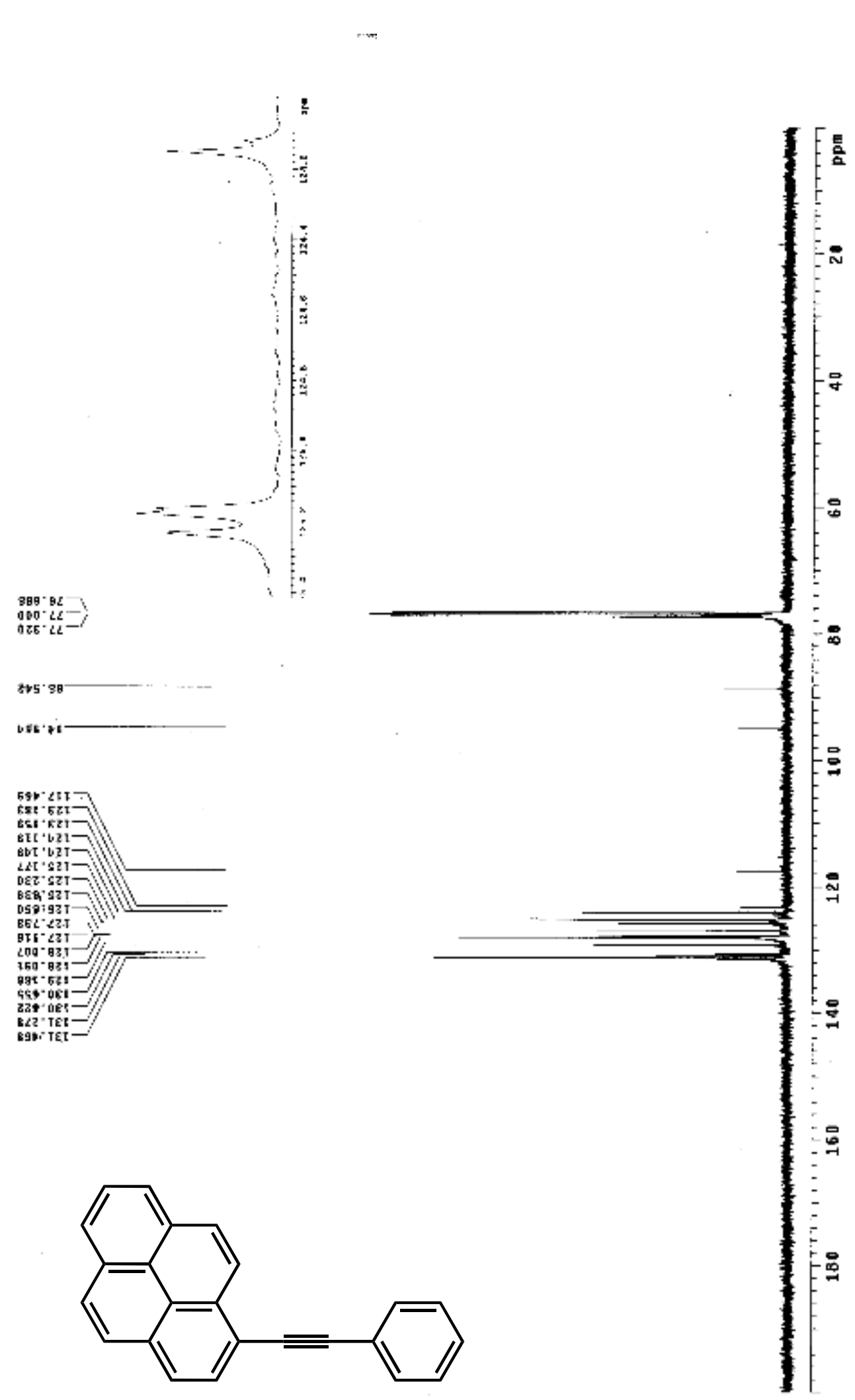


$50 v^{\circ} z z^{-}$
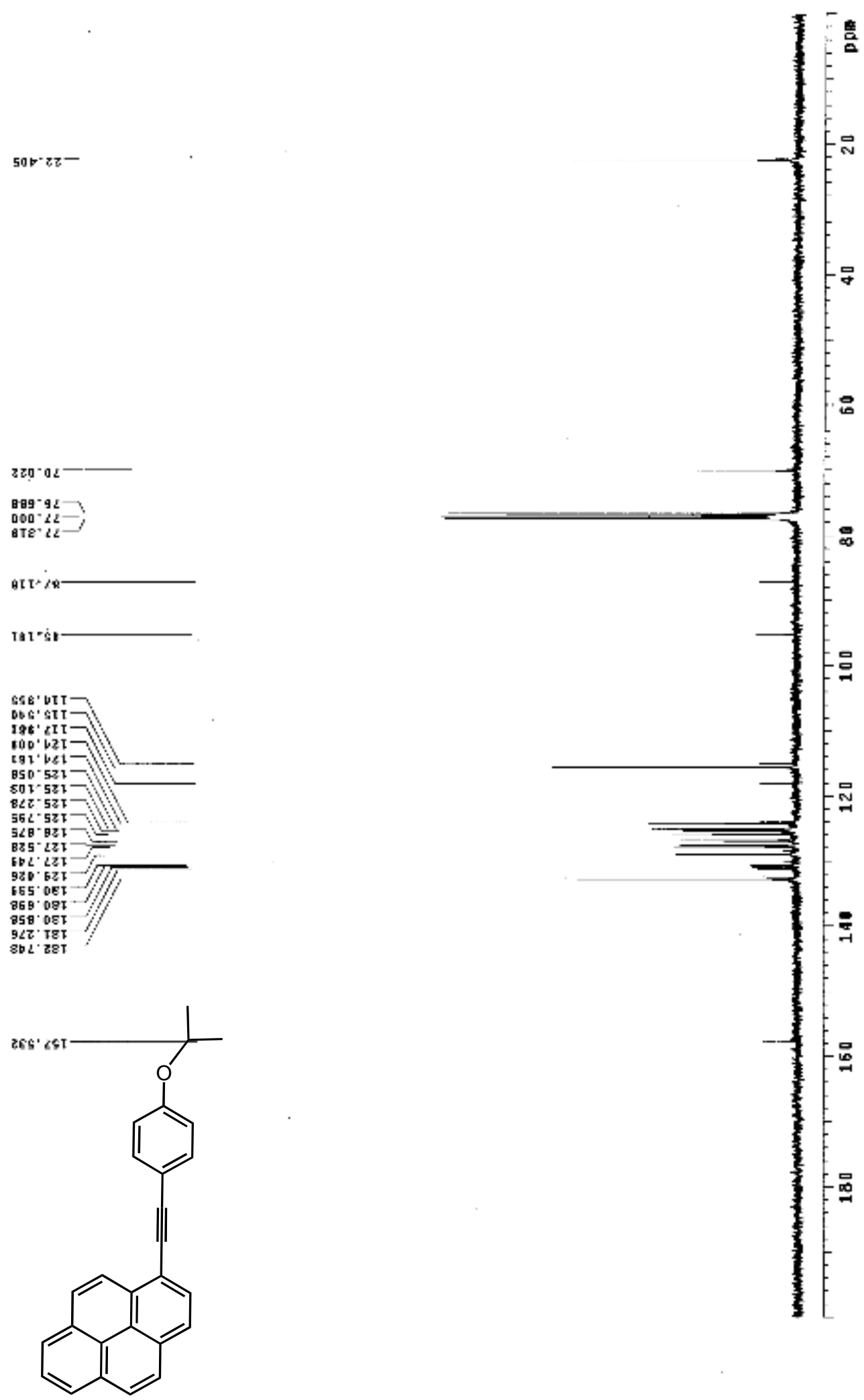

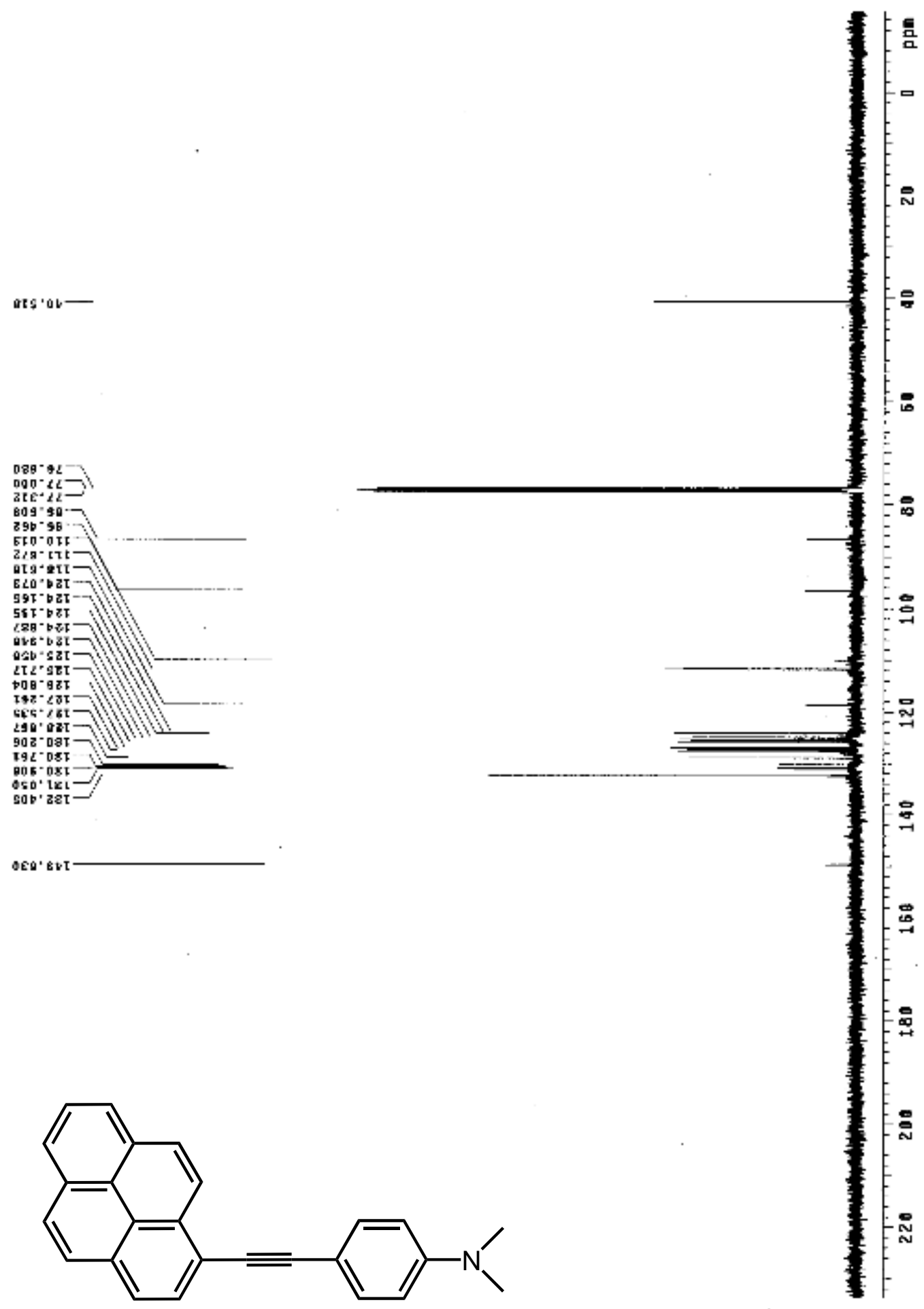


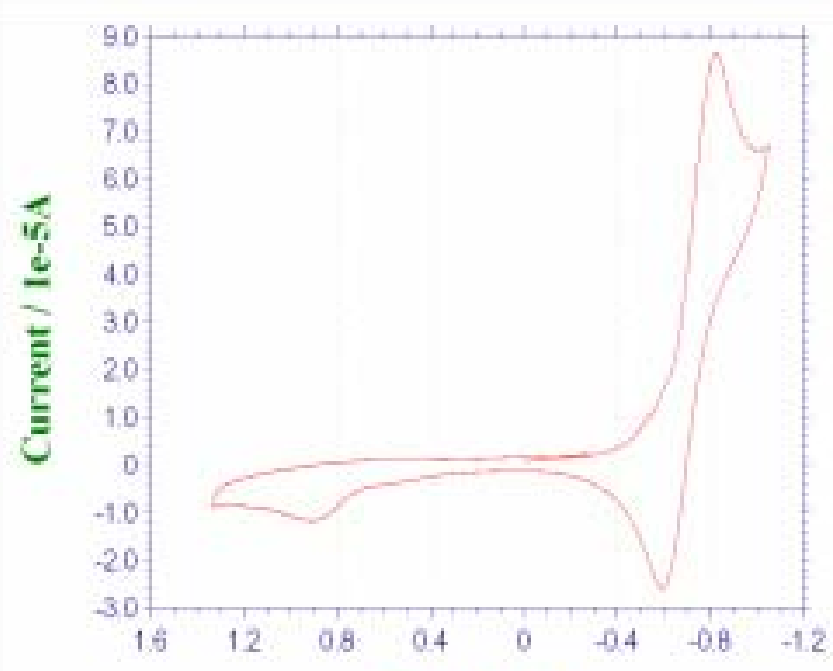

Fist. 15, 200518225

Fech CV

Fi. arfitakconces $2 \mathrm{bir}$

ish $E[V]=0$

$H$ on $E(V)=3$

los $P A y=4$

Scan Rate $\mid$ VIn $\mid=0$.

Segmern - 3

Smpi irterved [V] = D DC Qust $7 \mathrm{me}$ (a) $=2$

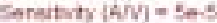

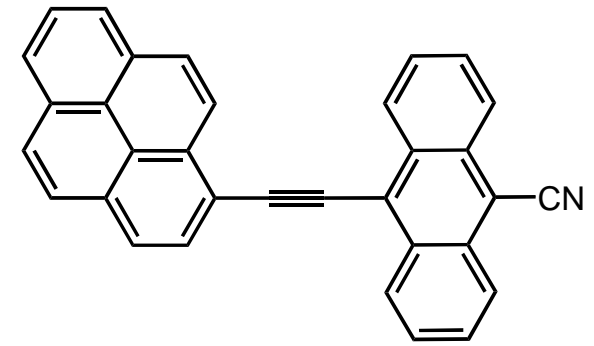

Potential / V

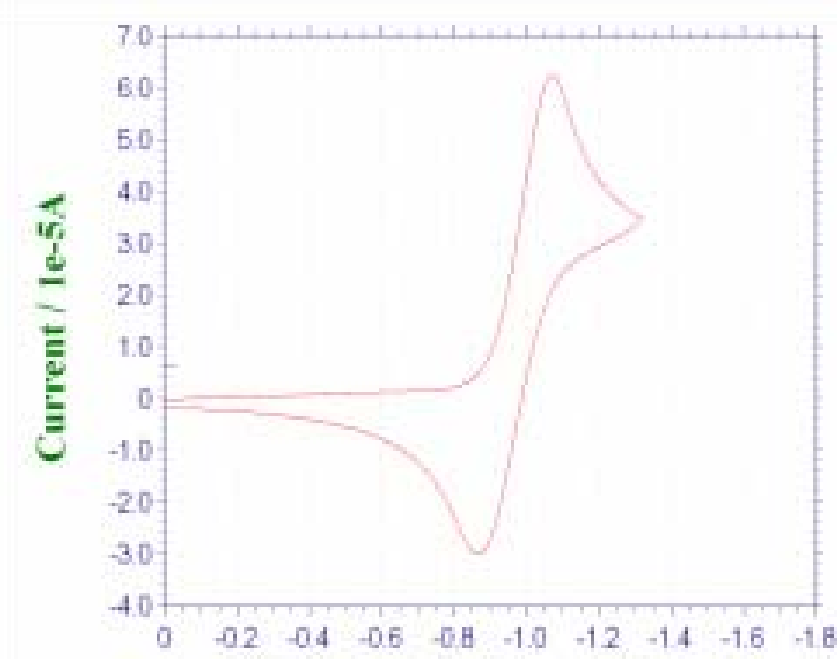

Mat. 8, 2005 1200.09

Tech: CV

Fie dibin

Int $E(n)=0$

High E $|V|=3$

Low $E(N)=-1$

$\log P$ Pi $=4$

Sean Rate (Vh) $=0.05$

Sepmant = 3

i iv] $=00$.

Gest Time $(s)=1$

Beminty |arvi a ze-5.

Potential / V vs $\mathrm{Ag} / \mathrm{AgCl}$
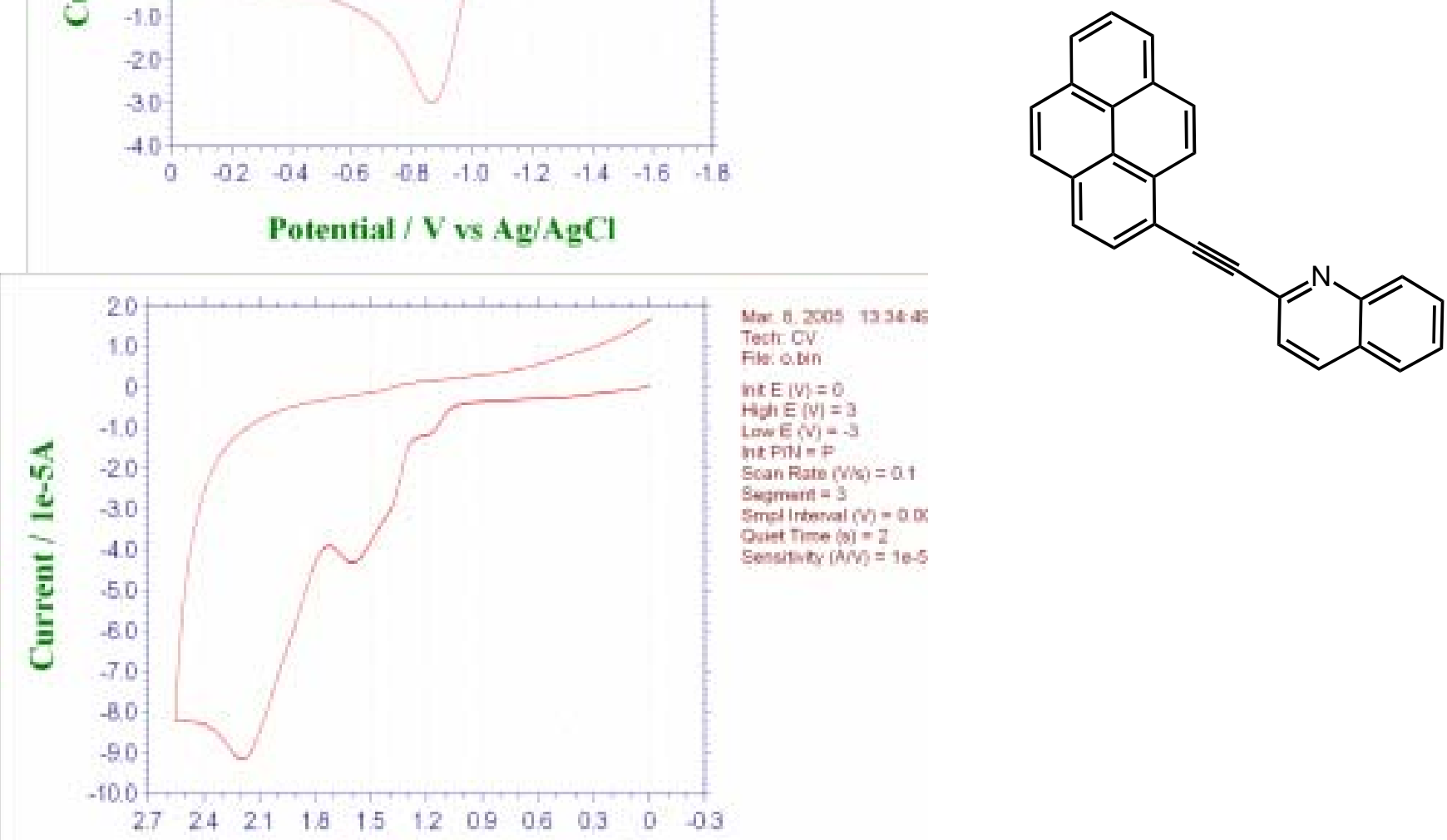

Potential / V vs Ag/AgCl 

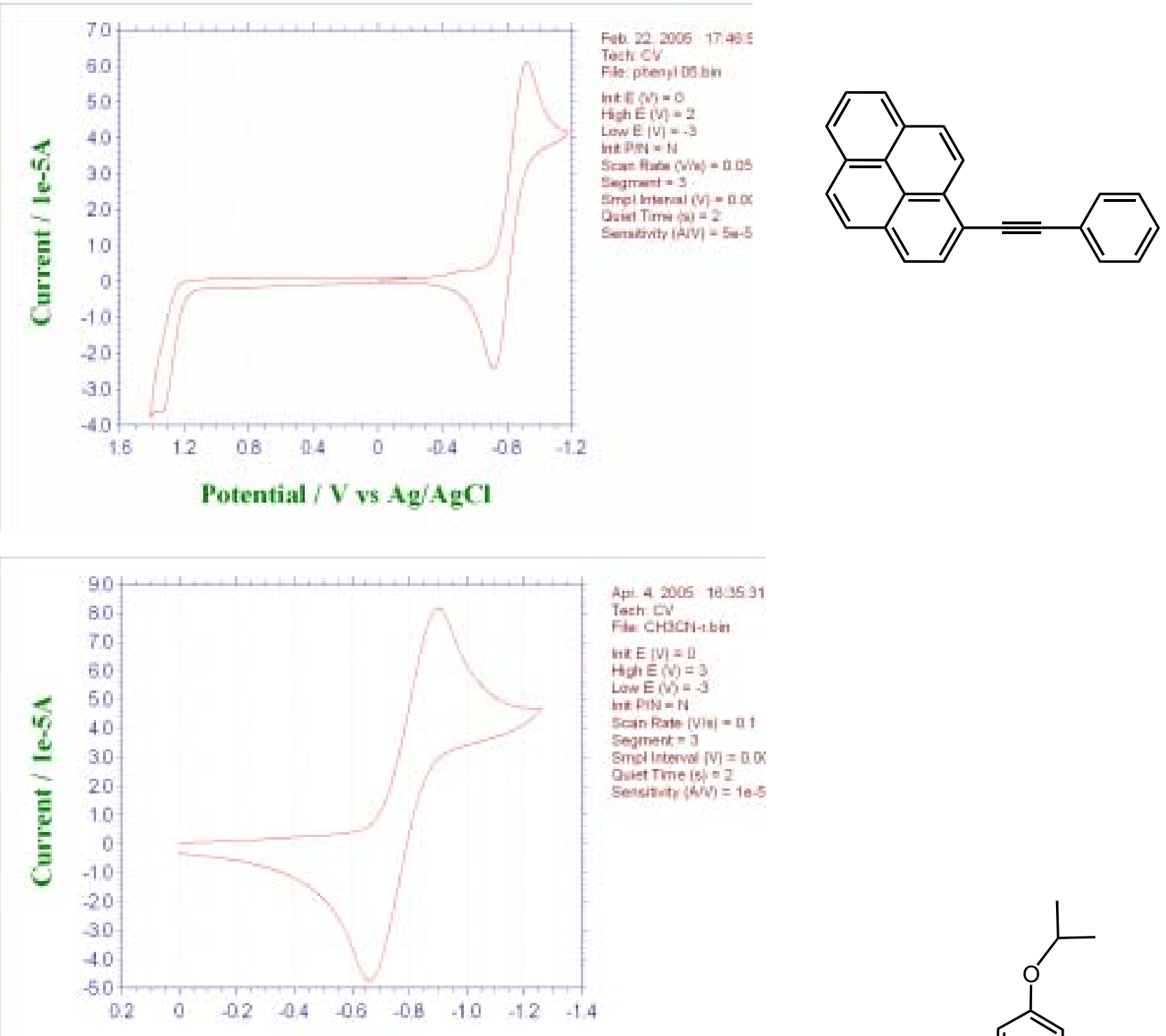

Api. 4 2005 1635 31 Tach 다

Fis CHacti-tem

he E $(V)=0$

HohE $(Y)=3$

Low $E M=-3$

he PiN $=\mathrm{N}$

Scan Rate $[$ Viei = 01

Segmert 73

Ginei interval $[V]=0.05$

onset thene is o o

Sesentrty $(\alpha V)=1 \mathrm{e}-5$

Potential / V
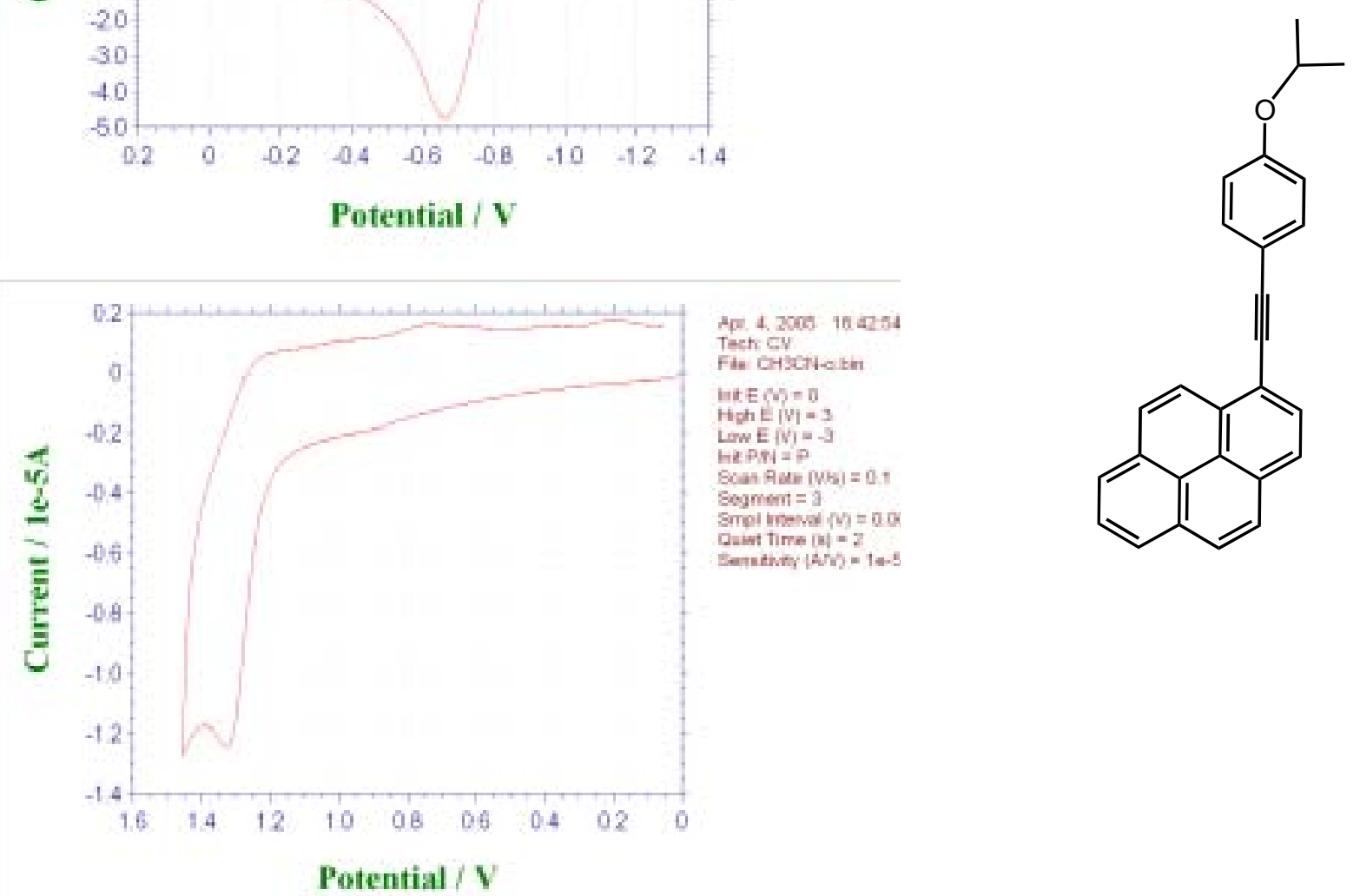

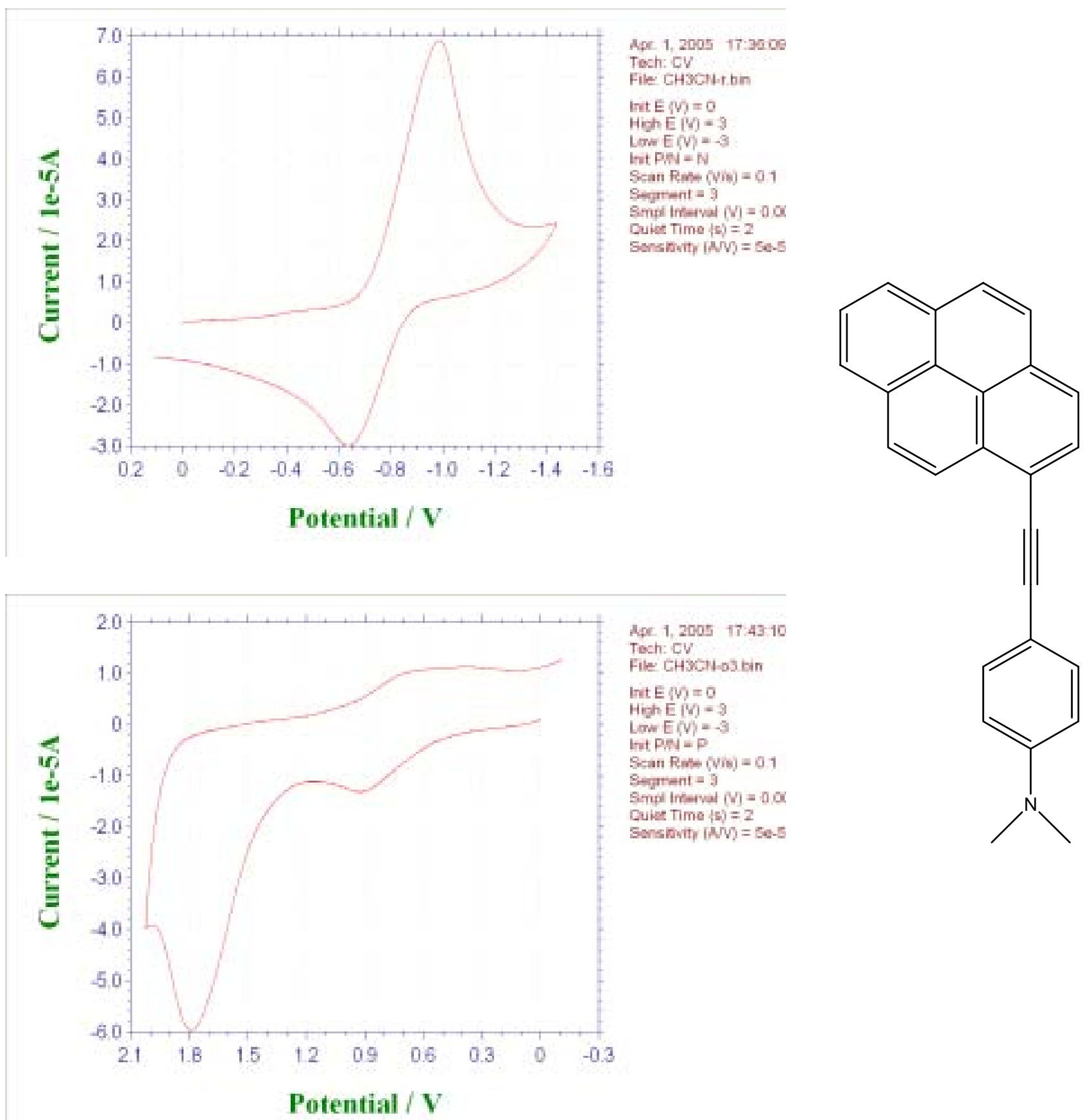

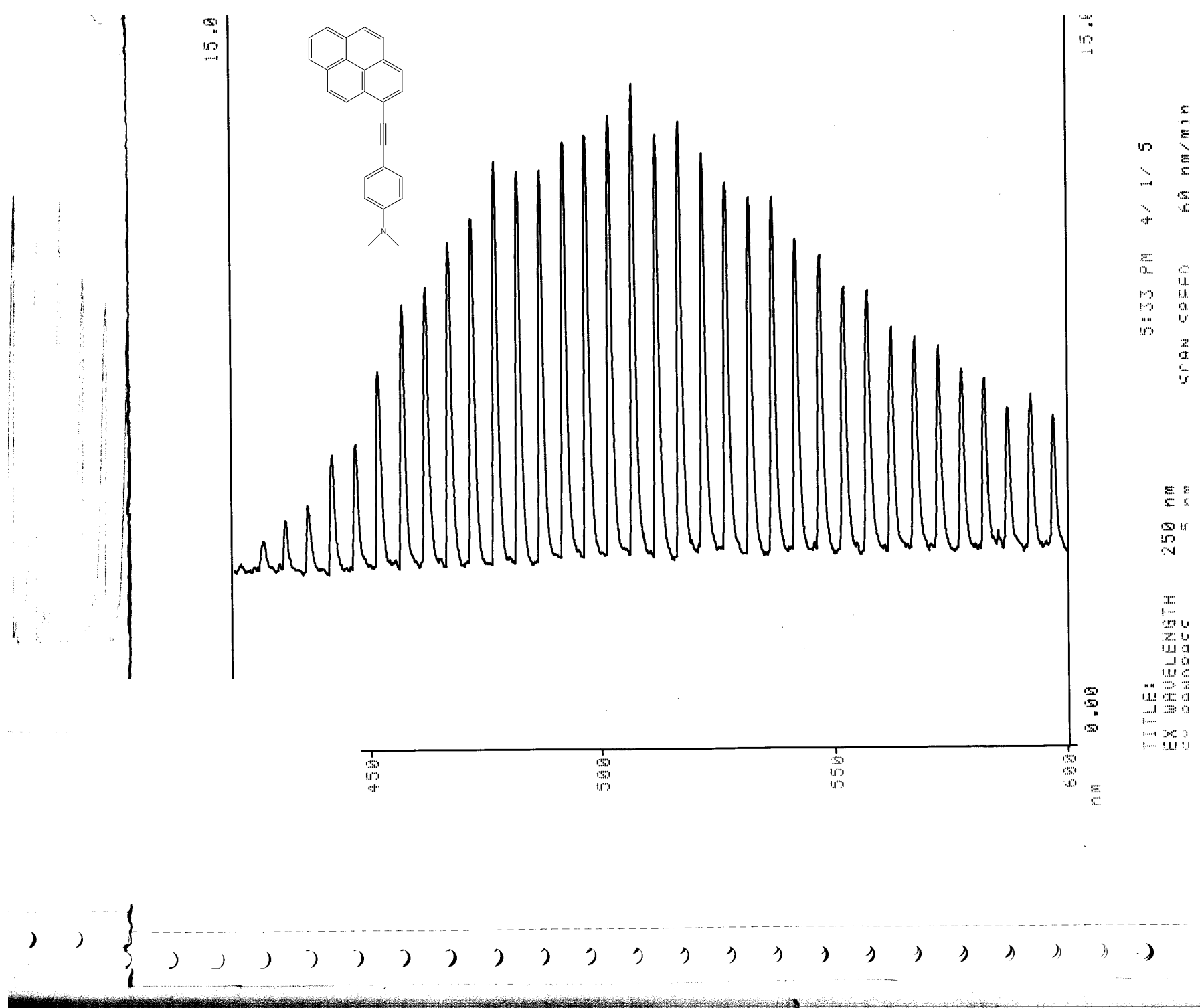


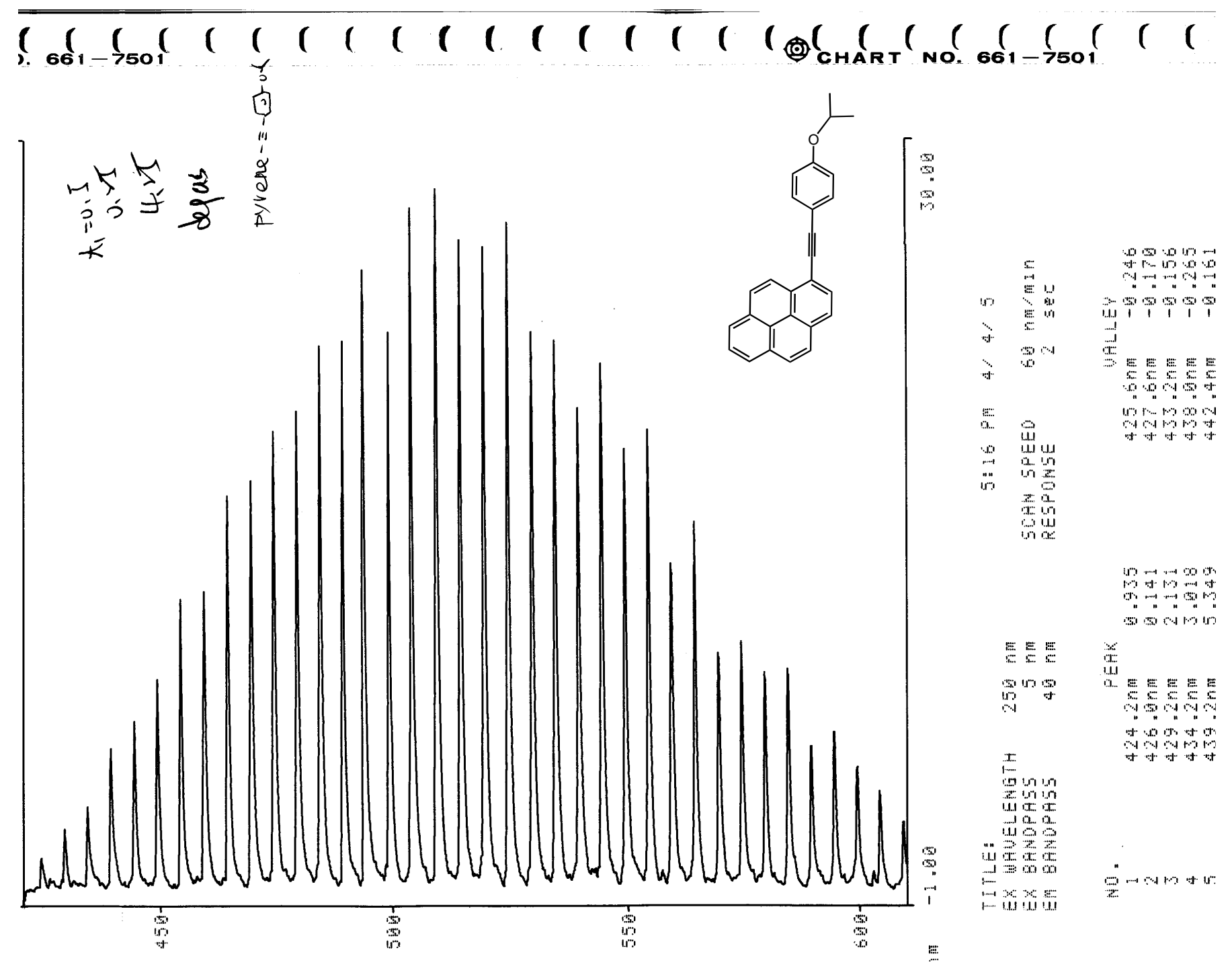


$\underline{\text { Solvatochromic data tables }}$

\section{ANEPy}

$\begin{array}{llrlrr}\text { solvent }(\in) & \begin{array}{l}\lambda_{\max } \text { abs } / \mathrm{nm} \\ {\left[v / \mathrm{cm}^{-1}\right]}\end{array} & \in_{\max } & \begin{array}{l}\lambda_{\max } \text { Flu } / \mathrm{nm} \\ {\left[v / \mathrm{cm}^{-1}\right]}\end{array} & \begin{array}{l}\text { stokes } \\ \text { shift } / \Delta v\left(\mathrm{~cm}^{-1}\right)\end{array} & \Phi^{\mathrm{Flu}} \\ \text { hexane(1.89) } & 472[21186] & 1.8 & 482[20747] & 439 & 1 \\ \text { dioxane(2.209) } & 478[21097] & 8 & 514.4[19440] & 1657 & 0.26 \\ \text { EA(6.02) } & 474[21097] & 1.7 & 517.6[19320] & 1777 & 0.87 \\ \operatorname{THF}(7.52) & 477[20964] & 2.4 & 534.1[18723] & 2241 & 0.84 \\ \text { CH2C12(9.08) } & 476[21108] & 9 & 540.5[18501] & 2607 & 0.22 \\ \text { CH3CN(37.5) } & 473[21142] & 1.7 & 554.9[18021] & 3121 & 0.45 \\ \operatorname{DMF}(38.3) & 481[20790] & 1.1 & 563.6[17743] & 3047 & 0.14 \\ \operatorname{DMSO}(48.9) & 485[20619] & 1.8 & 566.4[17655] & 2964 & 0.1\end{array}$

\section{QEPy}

\begin{tabular}{|c|c|c|c|c|c|}
\hline $\operatorname{solvent}(\in)$ & $\begin{array}{l}\lambda_{\max }{ }^{\mathrm{abs}} / \mathrm{nm} \\
{\left[\mathrm{v} / \mathrm{cm}^{-1}\right]}\end{array}$ & $\in_{\max }$ & $\begin{array}{l}\lambda_{\max }{ }^{\mathrm{Flu}} / \mathrm{nm} \\
{\left[\mathrm{v} / \mathrm{cm}^{-1}\right]}\end{array}$ & $\begin{array}{l}\text { stokes } \\
\text { shift } / \Delta v\left(\mathrm{~cm}^{-1}\right)\end{array}$ & $\Phi^{\mathrm{Flu}}$ \\
\hline hexane(1.89) & $397[25189]$ & 1.72 & $402[24876]$ & 313 & 0.99 \\
\hline dioxane(2.209) & $399[25063]$ & 1.2 & $415[24096]$ & 967 & 0.85 \\
\hline $\operatorname{EA}(6.02)$ & $396[25253]$ & 1.53 & $419[23866]$ & 1387 & 1 \\
\hline THF(7.52) & $398[25126]$ & 1.54 & $421[23753]$ & 1373 & 0.98 \\
\hline $\mathrm{CH} 2 \mathrm{Cl} 2(9.08)$ & $400[25000]$ & 2.05 & $436[22936]$ & 2064 & 1 \\
\hline $\mathrm{CH} 3 \mathrm{CN}(37.5)$ & $395[25316]$ & 1.32 & $444[22523]$ & 2793 & 0.83 \\
\hline $\operatorname{DMF}(38.3)$ & $399[25063]$ & 1.67 & $448[22321]$ & 2742 & 0.96 \\
\hline $\operatorname{DMSO}(48.9)$ & $401[24938]$ & 1.95 & $452[22124]$ & 2814 & 0.94 \\
\hline
\end{tabular}

\section{PhEPy}

$\operatorname{solvent}(\in)$

$\lambda_{\max }^{\text {abs }} / \mathrm{nm}$

$$
\begin{array}{ll}
\in_{\max } & \lambda_{\max } \mathrm{Flu} / \mathrm{nm} \\
& {\left[v / \mathrm{cm}^{-1}\right]}
\end{array}
$$

stokes

$\operatorname{shift} / \Delta v\left(\mathrm{~cm}^{-1}\right) \quad \Phi^{\mathrm{Flu}}$

hexane(1.89)

382 [26178]

$0.417389[25707]$

$471 \quad 0.62$

dioxane(2.209)

$384[26042]$

$0.419393[25445]$

596

1

EA(6.02)

382[26178]

$0.48 \quad 391[25575]$

603

0.94

$\operatorname{THF}(7.52)$

384 [26042]

$0.423 \quad 393[25445]$

596

0.92

$\mathrm{CH} 2 \mathrm{Cl} 2(9.08)$

385 [25974]

$0.388 \quad 394[25381]$

593

0.87

$\mathrm{CH} 3 \mathrm{CN}(37.5)$

$381[26247]$

$0.441392[25510]$

737

658

781

DMSO(48.9) 386[25907]

$0.444395[25316]$

$0.391398[25126]$

\section{IPPEPy}




\begin{tabular}{|c|c|c|c|c|c|}
\hline $\operatorname{solvent}(\in)$ & $\begin{array}{l}\lambda_{\max }^{\mathrm{abs}} / \mathrm{nm} \\
{\left[\mathrm{v} / \mathrm{cm}^{-1}\right]}\end{array}$ & $\in_{\max }$ & $\begin{array}{l}\lambda_{\max }{ }^{\mathrm{Flu}} / \mathrm{nm} \\
{\left[v / \mathrm{cm}^{-1}\right]}\end{array}$ & $\begin{array}{l}\text { stokes } \\
\text { shift } / \Delta v\left(\mathrm{~cm}^{-1}\right)\end{array}$ & $\Phi^{\mathrm{Flu}}$ \\
\hline hexane(1.89) & $386[25907]$ & 0.215 & $395[25316]$ & 590 & 0.79 \\
\hline dioxane(2.209) & $389[25707]$ & 0.207 & $403[24814]$ & 893 & 0.95 \\
\hline $\operatorname{EA}(6.02)$ & $386[25907]$ & 0.227 & $403[24814]$ & 1093 & 0.97 \\
\hline THF(7.52) & $388[25773]$ & 0.205 & $406[24631]$ & 1143 & 0.91 \\
\hline $\mathrm{CH} 2 \mathrm{Cl} 2(9.08)$ & $389[25707]$ & 0.267 & $412[24272]$ & 1435 & 0.91 \\
\hline $\mathrm{CH} 3 \mathrm{CN}(37.5)$ & $386[25907]$ & 0.222 & $420[23810]$ & 2097 & 0.98 \\
\hline $\operatorname{DMF}(38.3)$ & $391[25575]$ & 0.208 & $423[23641]$ & 1935 & 0.79 \\
\hline DMSO(48.9) & $394[25381]$ & 0.209 & $428[23364]$ & 2016 & 0.77 \\
\hline
\end{tabular}

\section{DMAEPy}

\begin{tabular}{|c|c|c|c|c|c|}
\hline $\operatorname{solvent}(\in)$ & $\begin{array}{l}\lambda_{\max }{ }^{a b s} / \mathrm{nm} \\
{\left[v / \mathrm{cm}^{-1}\right]}\end{array}$ & $\in_{\max }$ & $\begin{array}{l}\lambda_{\max }{ }^{\mathrm{Flu}} / \mathrm{nm} \\
{\left[\mathrm{v} / \mathrm{cm}^{-1}\right]}\end{array}$ & $\begin{array}{l}\text { stokes } \\
\text { shift } / \Delta v\left(\mathrm{~cm}^{-1}\right)\end{array}$ & $\Phi^{\text {Flu }}$ \\
\hline hexane(1.89) & $405[24691]$ & 0.493 & $421[23753]$ & 938 & 0.9 \\
\hline dioxane(2.209) & $405[24691]$ & 0.425 & $464[21552]$ & 3140 & 0.73 \\
\hline $\operatorname{EA}(6.02)$ & $401[24938]$ & 0.4 & $494[20243]$ & 4695 & 0.53 \\
\hline THF(7.52) & $404[24752]$ & 0.37 & $500[20000]$ & 4752 & 0.51 \\
\hline $\mathrm{CH} 2 \mathrm{Cl} 2(9.08)$ & $406[24631]$ & 0.449 & $497[20121]$ & 4510 & 0.61 \\
\hline $\mathrm{CH} 3 \mathrm{CN}(37.5)$ & $401[24938]$ & 0.477 & $540[18519]$ & 6419 & 0.23 \\
\hline $\operatorname{DMF}(38.3)$ & $407[24570]$ & 0.472 & $541[18484]$ & 6086 & 0.22 \\
\hline $\operatorname{DMSO}(48.9)$ & $410[24390]$ & 0.424 & $552[18116]$ & 6274 & 0.16 \\
\hline
\end{tabular}


(1) Lippert Mataga correlation
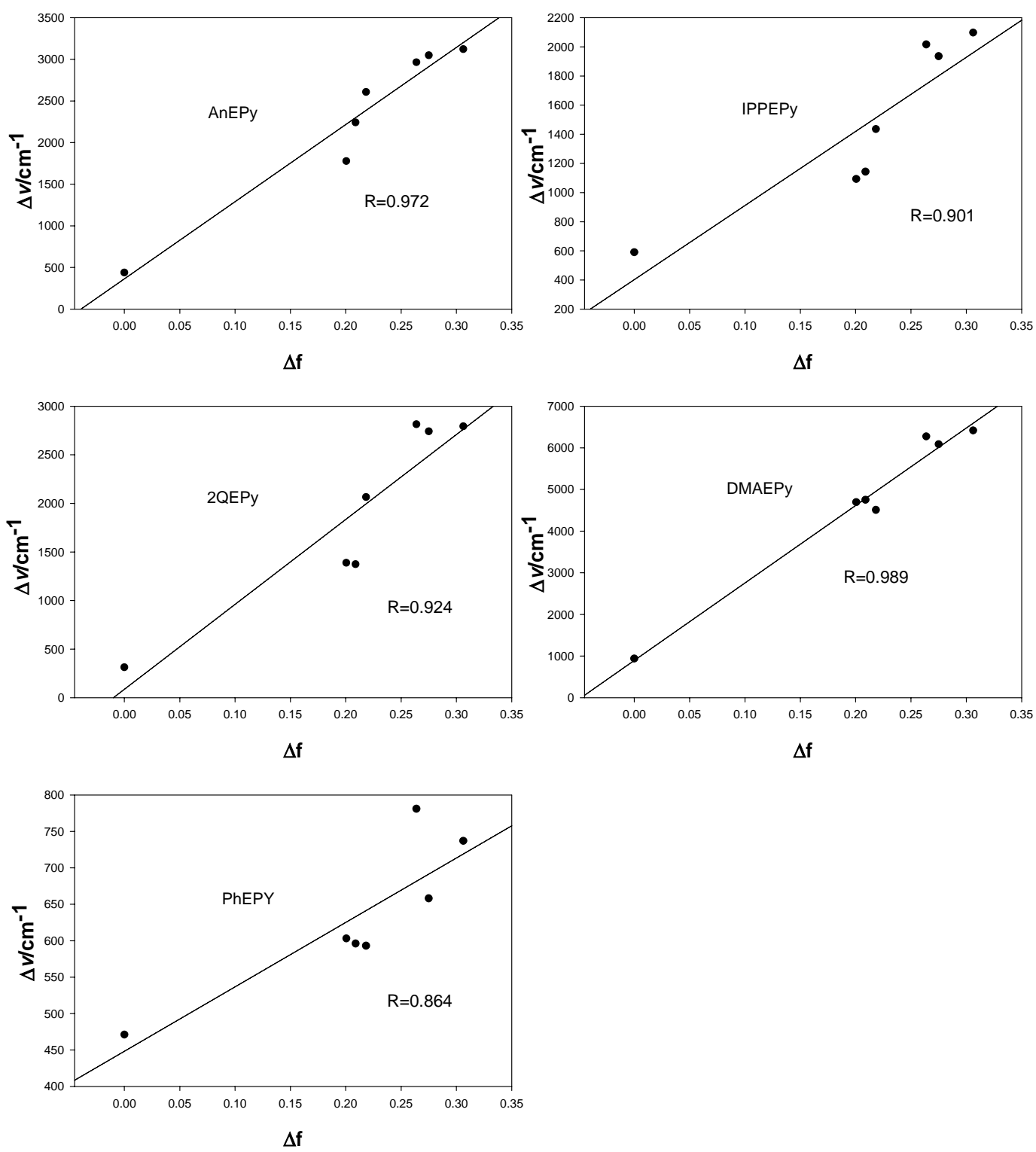
(2) Kamlet-Taft solvent polarity paramemter $\left(\pi^{*}\right)$ v.s. wavenumber of fluorescence
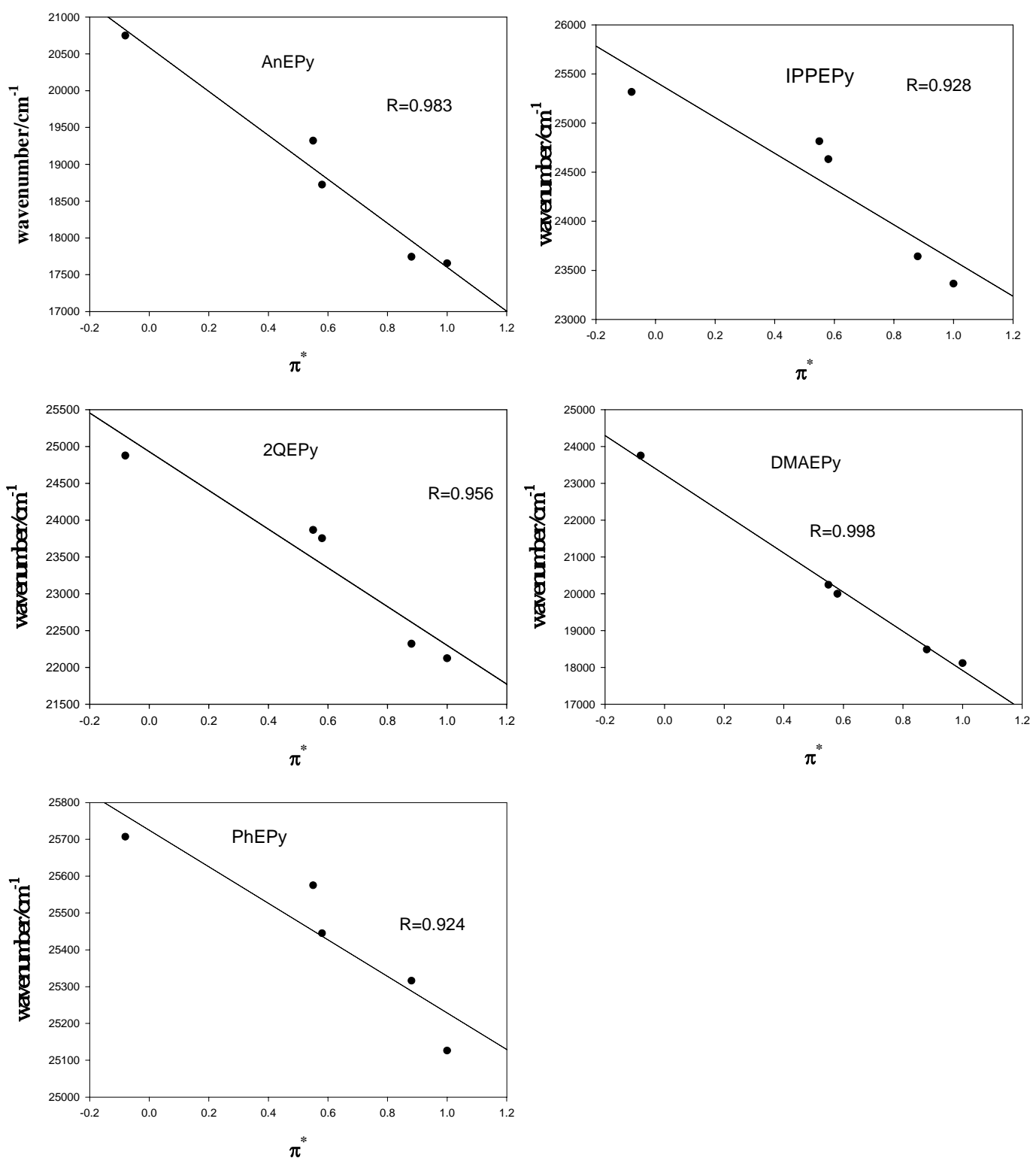
Concentration quenching of fluorescence in DMAEPy in hexane.

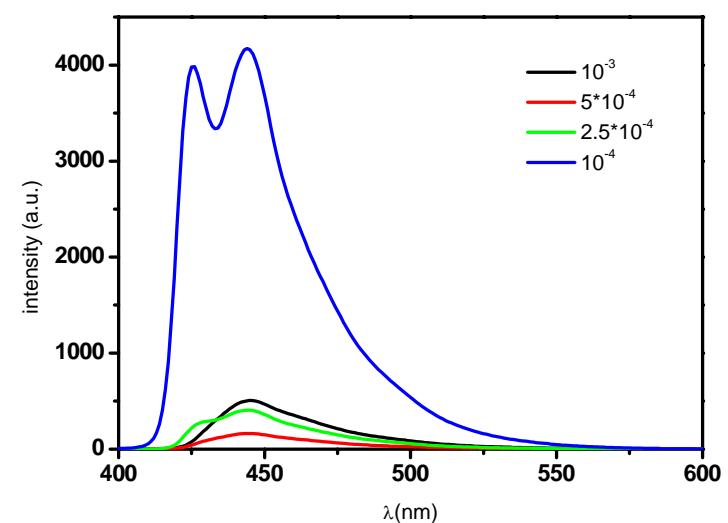

Concentration quenching and excimer formation of PhEPy in MeCN.

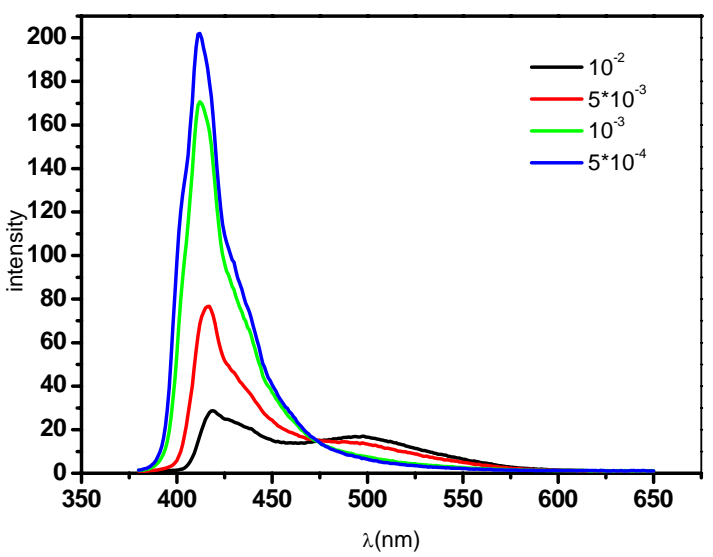

Concentration quenching of fluorescence of PhEPy in haxane.

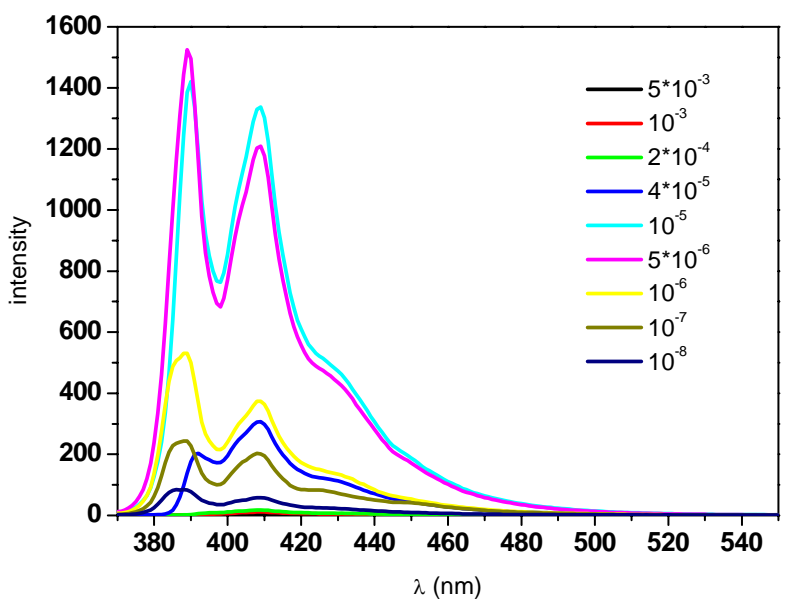


Concentration quenching of fluorescence of IPPEPy in MeCN.

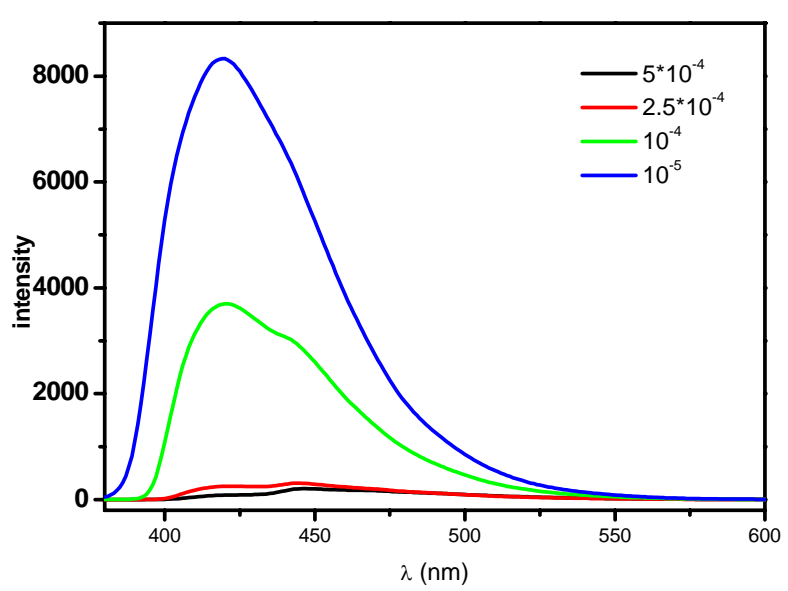

Concentration quenching of fluorescence of IPPEPy in hexane.

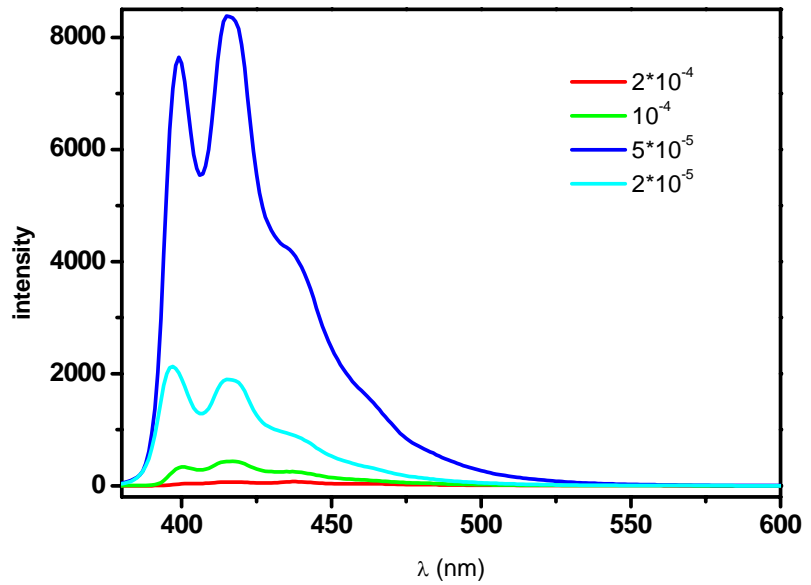




\section{Cartesian coordinates}

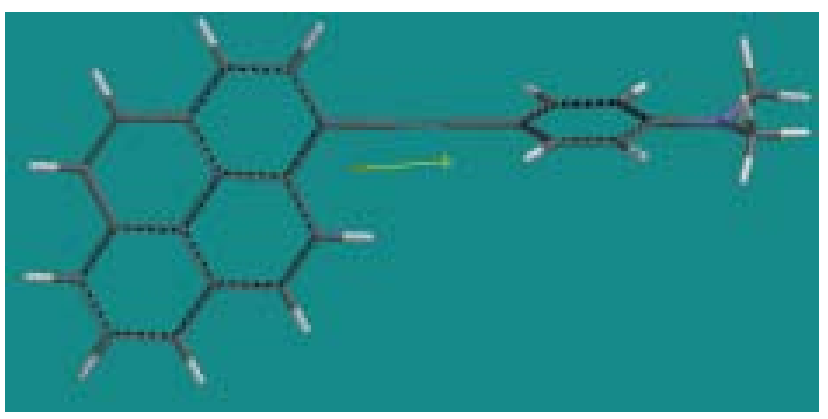

DMAEPy: energy: -1056.93 au. Imaginary frequencies: None

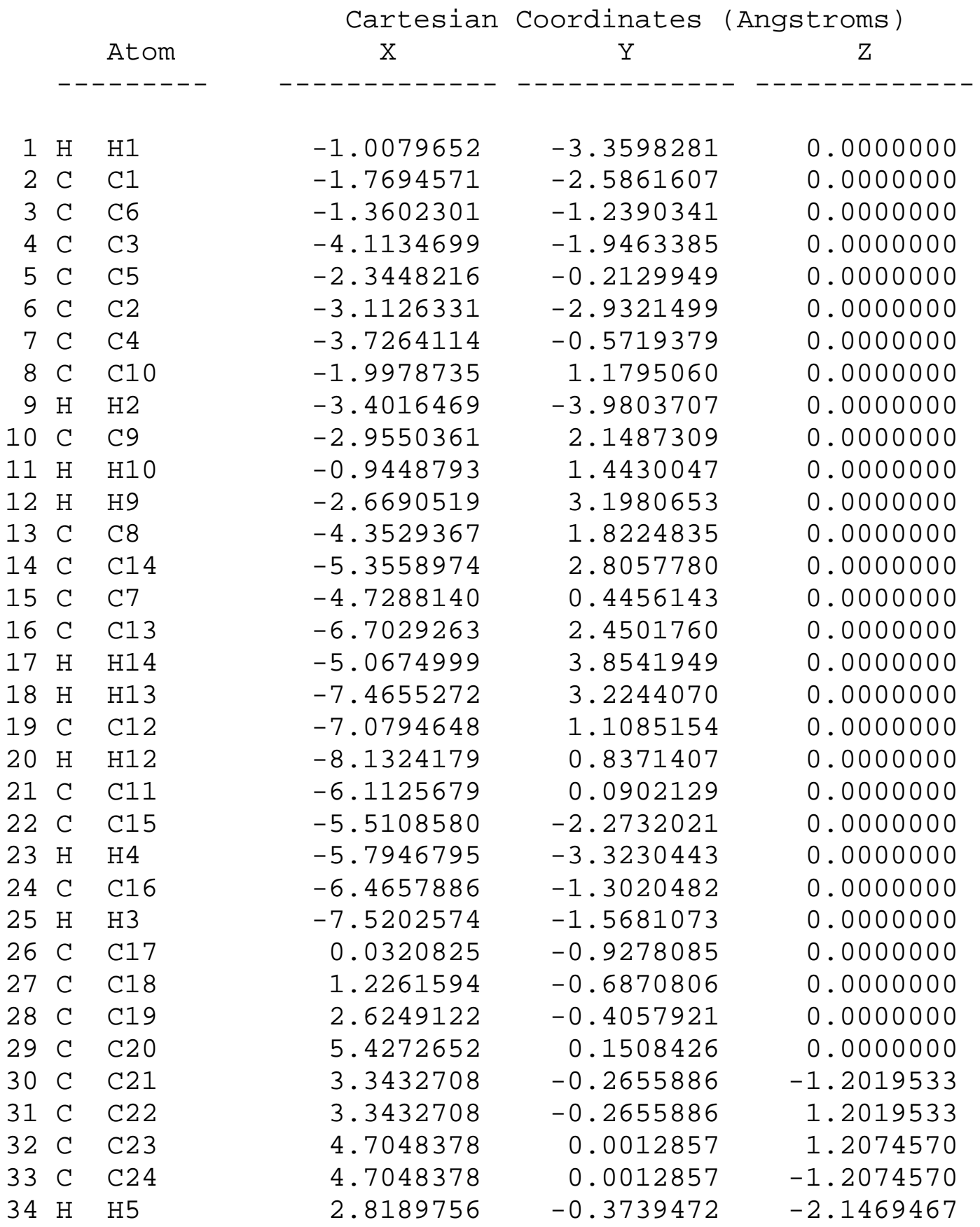




$\begin{array}{rllrrr}35 & \mathrm{H} & \mathrm{H} 6 & 2.8189756 & -0.3739472 & 2.1469467 \\ 36 & \mathrm{H} & \mathrm{H} 7 & 5.2078364 & 0.0947205 & 2.1624002 \\ 37 & \mathrm{H} & \mathrm{H} 8 & 5.2078364 & 0.0947205 & -2.1624002 \\ 38 & \mathrm{~N} & \mathrm{~N} 1 & 6.7831263 & 0.4406780 & 0.0000000 \\ 39 & \mathrm{C} & \mathrm{C} 25 & 7.5248928 & 0.4007018 & 1.2487989 \\ 40 & \mathrm{H} & \mathrm{H} 11 & 7.1270423 & 1.1271057 & 1.9680005 \\ 41 & \mathrm{H} & \mathrm{H} 16 & 8.5656983 & 0.6672088 & 1.0545668 \\ 42 & \mathrm{H} & \mathrm{H} 17 & 7.5072291 & -0.5932130 & 1.7238568 \\ 43 & \mathrm{C} & \mathrm{C} 26 & 7.5248928 & 0.4007018 & -1.2487989 \\ 44 & \mathrm{H} & \mathrm{H} 15 & 7.1270423 & 1.1271057 & -1.9680005 \\ 45 & \mathrm{H} & \mathrm{H} 18 & 7.5072291 & -0.5932130 & -1.7238568 \\ 46 & \mathrm{H} & \mathrm{H} 19 & 8.5656983 & 0.6672088 & -1.0545668\end{array}$

Point Group $=\mathrm{CS}$ Order $=1$ Nsymop $=2$

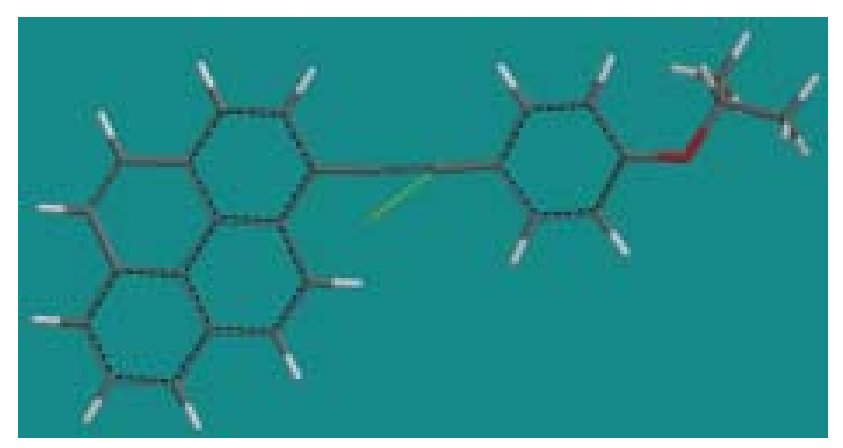

IPPEPy: Energy: -1116.15 au; imaginary frequencies: none.

\begin{tabular}{|c|c|c|c|c|c|}
\hline & & Atom & $\begin{array}{l}\text { Cartesian } \\
\quad \mathrm{x}\end{array}$ & $\begin{array}{c}\text { Coordinates } \\
Y\end{array}$ & $\begin{array}{c}\text { (Angstroms) } \\
Z\end{array}$ \\
\hline 1 & $\mathrm{H}$ & $\mathrm{H} 1$ & -1.2826174 & -2.9131757 & -1.1500279 \\
\hline 2 & $\mathrm{C}$ & $\mathrm{C} 1$ & -2.0944078 & -2.2457693 & -0.8779589 \\
\hline 3 & C & $\mathrm{C} 6$ & -1.7763016 & -0.9677483 & -0.3744605 \\
\hline 4 & $\mathrm{C}$ & $\mathrm{C} 3$ & -4.4756162 & -1.8051000 & -0.6856769 \\
\hline 5 & $\mathrm{C}$ & $\mathrm{C} 5$ & -2.8284571 & -0.0809386 & -0.0121241 \\
\hline 6 & $\mathrm{C}$ & $\mathrm{C} 2$ & -3.4095554 & -2.6544673 & -1.0285910 \\
\hline 7 & $\mathrm{C}$ & $\mathrm{C} 4$ & -4.1818905 & -0.5053713 & -0.1717964 \\
\hline 8 & $\mathrm{C}$ & C10 & -2.5775300 & 1.2314530 & 0.5091021 \\
\hline 9 & $\mathrm{H}$ & $\mathrm{H} 2$ & -3.6264597 & -3.6460063 & -1.4185454 \\
\hline 10 & $\mathrm{C}$ & $\mathrm{C} 9$ & -3.5988583 & 2.0676847 & 0.8464622 \\
\hline 11 & $\mathrm{H}$ & $\mathrm{H} 10$ & -1.5452675 & 1.5448042 & 0.6291492 \\
\hline 12 & $\mathrm{H}$ & H9 & -3.3866923 & 3.0593778 & 1.2389343 \\
\hline 13 & $\mathrm{C}$ & $\mathrm{C} 8$ & -4.9708724 & 1.6738611 & 0.6962702 \\
\hline 14 & $\mathrm{C}$ & C14 & -6.0389676 & 2.5207684 & 1.0356035 \\
\hline 15 & $\mathrm{C}$ & C7 & -5.2518080 & 0.3726707 & 0.1811703 \\
\hline 16 & $\mathrm{C}$ & $\mathrm{C} 13$ & -7.3583110 & 2.1033595 & 0.8741722 \\
\hline 17 & $\mathrm{H}$ & $\mathrm{H} 14$ & -5.8237484 & 3.5121765 & 1.4270151 \\
\hline 18 & $\mathrm{H}$ & $\mathrm{H} 13$ & -8.1720838 & 2.7720002 & 1.1417843 \\
\hline 19 & $\mathrm{C}$ & $\mathrm{C} 12$ & -7.6422380 & 0.8350041 & 0.3716772 \\
\hline
\end{tabular}




$\begin{array}{rllrrr}20 & \text { H } & \text { H12 } & -8.6741375 & 0.5159342 & 0.2469058 \\ 21 & \text { C } & \text { C11 } & -6.6079371 & -0.0470928 & 0.0192903 \\ 22 & \text { C } & \text { C15 } & -5.8463091 & -2.1997475 & -0.8355294 \\ 23 & \text { H } & \text { H4 } & -6.0560566 & -3.1923497 & -1.2269017 \\ 24 & \text { C } & \text { C16 } & -6.8659264 & -1.3607809 & -0.5005410 \\ 25 & \text { H } & \text { H3 } & -7.8999104 & -1.6745731 & -0.6221882 \\ 26 & \text { C } & \text { C17 } & -0.4101708 & -0.5973322 & -0.2479751 \\ 27 & \text { C } & \text { C18 } & 0.7743628 & -0.3235296 & -0.1634303 \\ 28 & \text { C } & \text { C19 } & 2.1659664 & -0.0383856 & -0.0861954 \\ 29 & \text { C } & \text { C20 } & 4.9383563 & 0.5014910 & 0.0449859 \\ 30 & \text { C } & \text { C21 } & 3.1126100 & -0.9702553 & -0.5488776 \\ 31 & \text { C } & \text { C22 } & 2.6451469 & 1.1765284 & 0.4506131 \\ 32 & \text { C } & \text { C23 } & 4.0034487 & 1.4393976 & 0.5154763 \\ 33 & \text { C } & \text { C24 } & 4.4795183 & -0.7127989 & -0.4857916 \\ 34 & \text { H } & \text { H5 } & 2.7668682 & -1.9122548 & -0.9635886 \\ 35 & \text { H } & \text { H6 } & 1.9356325 & 1.9127530 & 0.8158165 \\ 36 & \text { H } & \text { H7 } & 4.3741729 & 2.3740022 & 0.9245694 \\ 37 & \text { H } & \text { H8 } & 5.1707518 & -1.4626170 & -0.8517457 \\ 38 & \text { O } & \text { O1 } & 6.2423680 & 0.8827959 & 0.1378179 \\ 39 & \text { C } & \text { C25 } & 7.3024343 & -0.0010956 & -0.2775076 \\ 40 & \text { H } & \text { H15 } & 7.0063691 & -0.4945329 & -1.2130310 \\ 41 & \text { C } & \text { C26 } & 8.5027965 & 0.8984083 & -0.5520855 \\ 42 & \text { H } & \text { H11 } & 8.7939998 & 1.4345258 & 0.3576107 \\ 43 & \text { H } & \text { H17 } & 8.2629836 & 1.6349566 & -1.3250114 \\ 44 & \text { H } & \text { H18 } & 9.3559531 & 0.3012135 & -0.8914032 \\ 45 & \text { C } & \text { C27 } & 7.5842716 & -1.0438828 & 0.8048051 \\ 46 & \text { H } & \text { H16 } & 7.8984837 & -0.5476008 & 1.7295475 \\ 47 & \text { H } & \text { H19 } & 8.3863356 & -1.7195610 & 0.4859776 \\ 48 & \text { H } & \text { H20 } & 6.6993008 & -1.6481996 & 1.0262279\end{array}$

Point Group $=\mathrm{C} 1$ Order $=1$ Nsymop $=1$

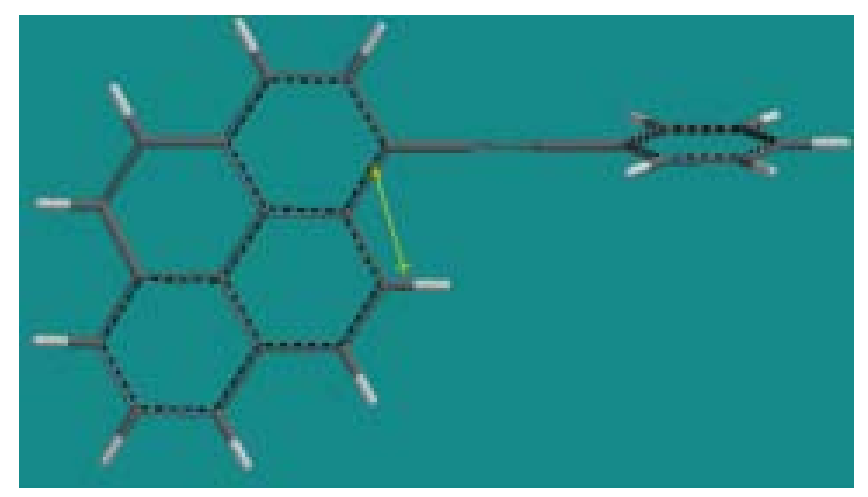

PhEPy: Energy: -922.98 au; imaginary frequencies: none

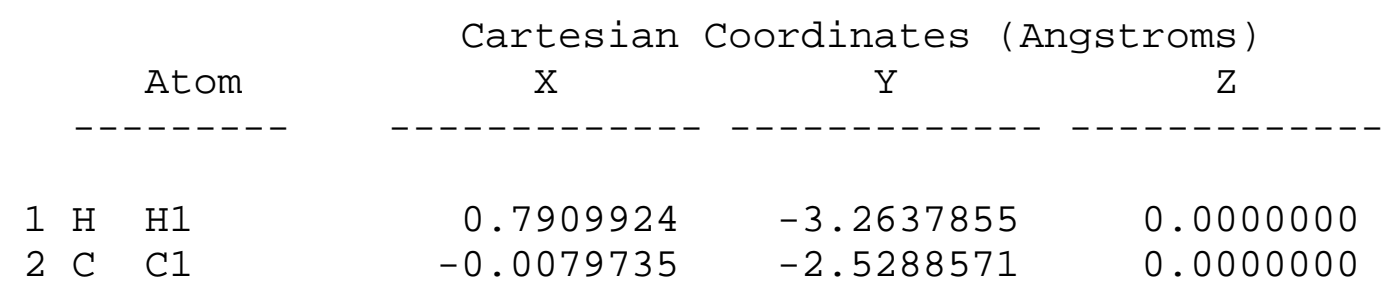




$\begin{array}{rll}3 & \mathrm{C} & \mathrm{C} 6 \\ 4 & \mathrm{C} & \mathrm{C} 3 \\ 5 & \mathrm{C} & \mathrm{C} 5 \\ 6 & \mathrm{C} & \mathrm{C} 2 \\ 7 & \mathrm{C} & \mathrm{C} 4 \\ 8 & \mathrm{C} & \mathrm{C} 10 \\ 9 & \mathrm{H} & \mathrm{H} 2 \\ 10 & \mathrm{C} & \mathrm{C} 9 \\ 11 & \mathrm{H} & \mathrm{H} 10 \\ 12 & \mathrm{H} & \mathrm{H} 9 \\ 13 & \mathrm{C} & \mathrm{C} 8 \\ 14 & \mathrm{C} & \mathrm{C} 14 \\ 15 & \mathrm{C} & \mathrm{C} 7 \\ 16 & \mathrm{C} & \mathrm{C} 13 \\ 17 & \mathrm{H} & \mathrm{H} 14 \\ 18 & \mathrm{H} & \mathrm{H} 13 \\ 19 & \mathrm{C} & \mathrm{C} 12 \\ 20 & \mathrm{H} & \mathrm{H} 12 \\ 21 & \mathrm{C} & \mathrm{C} 11 \\ 22 & \mathrm{C} & \mathrm{C} 15 \\ 23 & \mathrm{H} & \mathrm{H} 4 \\ 24 & \mathrm{C} & \mathrm{C} 16 \\ 25 & \mathrm{H} & \mathrm{H} 3 \\ 26 & \mathrm{C} & \mathrm{C} 17 \\ 27 & \mathrm{C} & \mathrm{C} 18 \\ 28 & \mathrm{C} & \mathrm{C} 19 \\ 29 & \mathrm{C} & \mathrm{C} 20 \\ 30 & \mathrm{C} & \mathrm{C} 21 \\ 31 & \mathrm{C} & \mathrm{C} 22 \\ 32 & \mathrm{C} & \mathrm{C} 23 \\ 33 & \mathrm{C} & \mathrm{C} 24 \\ 34 & \mathrm{H} & \mathrm{H} 5 \\ 35 & \mathrm{H} & \mathrm{H} 6 \\ 36 & \mathrm{H} & \mathrm{H} 7 \\ 37 & \mathrm{H} & \mathrm{H} 8 \\ 38 & \mathrm{H} & \mathrm{H} 20\end{array}$

0.3309171
-2.3812786
-0.7022714
-1.3322319
-2.0636179
-0.4251090
-1.5675678
-1.4298907
0.6125989
-1.1975890
-2.8092846
-3.8604223
-3.1158843
-5.1878376
-3.6257413
-5.9882255
-5.4966156
-6.5345367
-4.4795553
-3.7604003
-3.9906693
-4.7624930
-5.8022970
1.7046223
2.8794021
4.2587648
6.9692693
4.9497849
4.9497849
6.2931350
6.2931350
4.4219006
4.4219006
6.8139463
6.8139463
8.0173920

$-1.1626791$

$-2.0068545$

$-0.1861767$

$-2.9413045$

$-0.6143809$

1.2220616

$-4.0026544$

2.1417403

1.5403423

3. 2041451

1. 7453828

2. 6768785

0.3513455

2. 2540405

3. 7384861

2.9890379

0.8950741

0.5707652

$-0.0730544$

$-2.4037362$

$-3.4664738$

$-1.4813458$

$-1.7997323$

$-0.7780466$

$-0.4597414$

$-0.0830053$

0.6585267

0.1065223

0.1065223

0.4737435

0.4737435

$-0.0374140$

$-0.0374140$

0.6164750

0.6164750

0.9453483
0.0000000

0.0000000

0.0000000

0.0000000

0.0000000

0.0000000

0.0000000

0.0000000

0.0000000

0.0000000

0.0000000

0.0000000

0.0000000

0.0000000

0.0000000

0.0000000

0.0000000

0.0000000

0.0000000

0.0000000

0.0000000

0.0000000

0.0000000

0.0000000

0.0000000

0.0000000

0.0000000

$-1.2123095$

1. 2123095

1.2079096

$-1.2079096$

$-2.1501034$

2. 1501034

2.1509854

$-2.1509854$

0.0000000

Point Group $=\mathrm{CS}$ Order $=1$ Nsymop $=2$

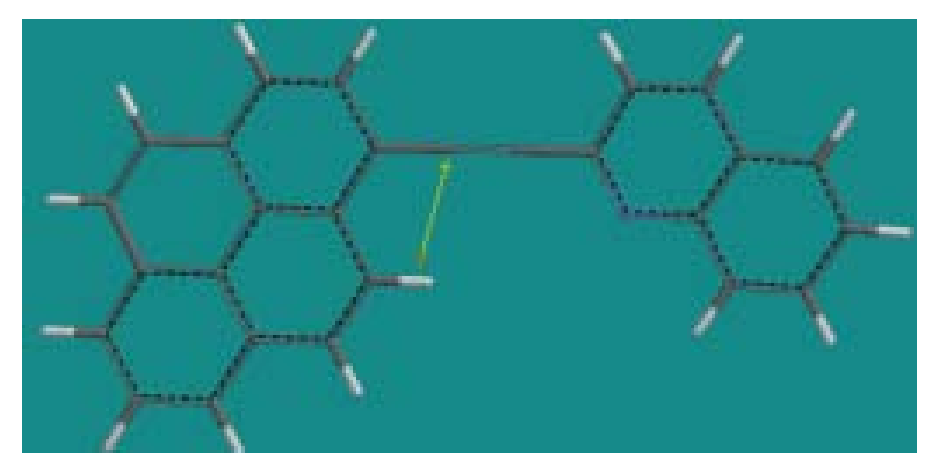


2QEPy: Energy: -1092.67; imaginary frequencies: none

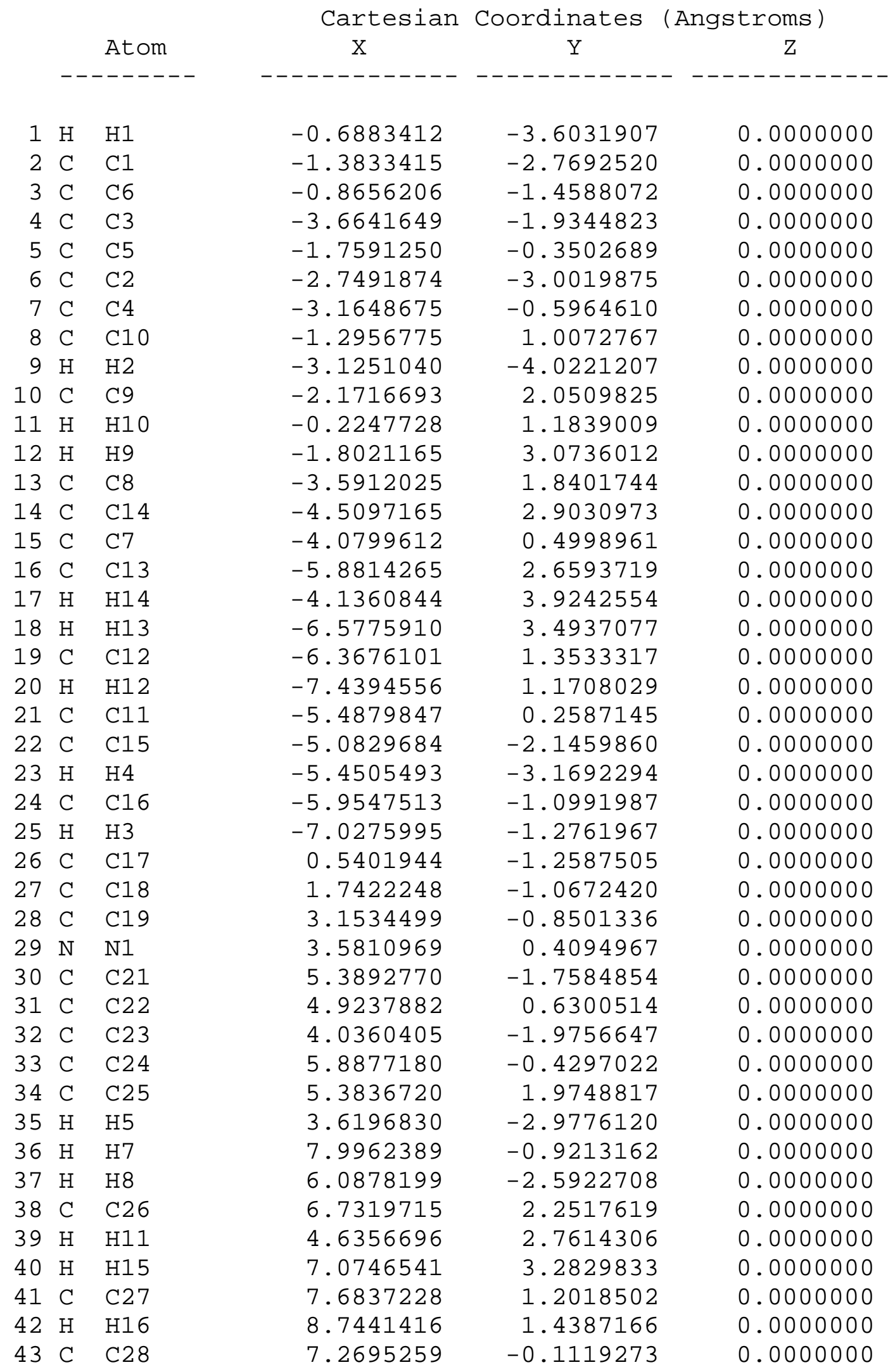

Point Group $=\mathrm{CS}$ Order $=1$ Nsymop $=2$ 


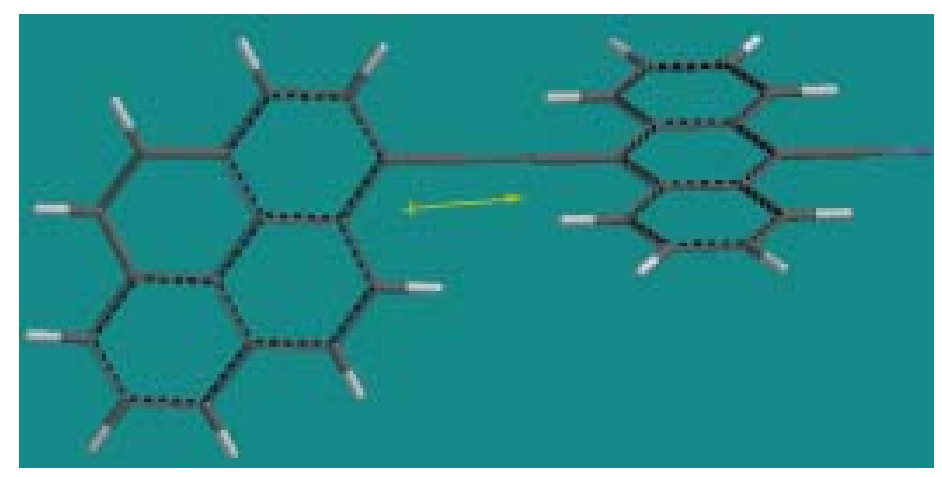

ANEPy: Energy: -1322.51 au; imaginary frequencies: none

\begin{tabular}{|c|c|c|c|c|c|}
\hline & & Atom & $\begin{array}{l}\text { Cartesian } \\
\quad \mathrm{x}\end{array}$ & $\begin{array}{c}\text { Coordinates } \\
Y\end{array}$ & $\begin{array}{c}\text { (Angstroms) } \\
Z\end{array}$ \\
\hline 1 & $\mathrm{H}$ & $\mathrm{H} 1$ & 0.8264408 & 0.0038100 & 3.3024396 \\
\hline 2 & $\mathrm{C}$ & $\mathrm{C} 1$ & 1.6052407 & 0.0029903 & 2.5461852 \\
\hline 3 & $\mathrm{C}$ & C6 & 1.2319749 & 0.0014818 & 1.1888419 \\
\hline 4 & $\mathrm{C}$ & $\mathrm{C} 3$ & 3.9628883 & 0.0023507 & 1.9588292 \\
\hline 5 & $\mathrm{C}$ & $\mathrm{C} 5$ & 2.2362539 & 0.0002803 & 0.1829369 \\
\hline 6 & $\mathrm{C}$ & $\mathrm{C} 2$ & 2.9399155 & 0.0035164 & 2.9222721 \\
\hline 7 & $\mathrm{C}$ & $\mathrm{C} 4$ & 3.6085763 & 0.0008293 & 0.5751791 \\
\hline 8 & $\mathrm{C}$ & $\mathrm{C} 10$ & 1.9212240 & -0.0012793 & -1.2171739 \\
\hline 9 & $\mathrm{H}$ & $\mathrm{H} 2$ & 3.2042972 & 0.0046228 & 3.9765866 \\
\hline 10 & $\mathrm{C}$ & $\mathrm{C} 9$ & 2.9015211 & -0.0025163 & -2.1631714 \\
\hline 11 & $\mathrm{H}$ & $\mathrm{H} 10$ & 0.8758273 & -0.0016446 & -1.5099259 \\
\hline 12 & $\mathrm{H}$ & H9 & 2.6401191 & -0.0036478 & -3.2187167 \\
\hline 13 & $\mathrm{C}$ & $\mathrm{C} 8$ & 4.2909237 & -0.0020311 & -1.8037334 \\
\hline 14 & $\mathrm{C}$ & C14 & 5.3166368 & -0.0032666 & -2.7631566 \\
\hline 15 & $\mathrm{C}$ & C7 & 4.6343055 & -0.0004618 & -0.4185264 \\
\hline 16 & $\mathrm{C}$ & C13 & 6.6548771 & -0.0027313 & -2.3759394 \\
\hline 17 & $\mathrm{H}$ & $\mathrm{H} 14$ & 5.0534868 & -0.0044374 & -3.8179870 \\
\hline 18 & $\mathrm{H}$ & $\mathrm{H} 13$ & 7.4353903 & -0.0036314 & -3.1318179 \\
\hline 19 & $\mathrm{C}$ & $\mathrm{C} 12$ & 6.9994319 & -0.0012047 & -1.0256093 \\
\hline 20 & $\mathrm{H}$ & $\mathrm{H} 12$ & 8.0455631 & -0.0007884 & -0.7293354 \\
\hline 21 & $\mathrm{C}$ & $\mathrm{C} 11$ & 6.0087155 & 0.0000565 & -0.0304892 \\
\hline 22 & $\mathrm{C}$ & C15 & 5.3521309 & 0.0028581 & 2.3188269 \\
\hline 23 & $\mathrm{H}$ & H 4 & 5.6111211 & 0.0040126 & 3.3748189 \\
\hline 24 & $\mathrm{C}$ & C16 & 6.3290584 & 0.0016440 & 1.3696386 \\
\hline 25 & $\mathrm{H}$ & H3 & 7.3768273 & 0.0020175 & 1.6604933 \\
\hline 26 & $\mathrm{C}$ & C17 & -0.1522461 & 0.0011761 & 0.8447495 \\
\hline 27 & $\mathrm{C}$ & C18 & -1.3397026 & 0.0005868 & 0.5706147 \\
\hline 28 & $\mathrm{H}$ & H5 & -2.8975320 & -4.6140054 & 0.2058999 \\
\hline 29 & $\mathrm{C}$ & C19 & -3.4240422 & -3.6700155 & 0.0978186 \\
\hline 30 & $\mathrm{H}$ & H 6 & -1.6862570 & -2.4814631 & 0.4690975 \\
\hline
\end{tabular}




$\begin{array}{rlllrr}31 & \text { C } & \text { C20 } & -2.7476704 & -2.4874912 & 0.2452698 \\ 32 & \text { C } & \text { C21 } & -5.4941560 & -2.4878295 & -0.3372888 \\ 33 & \text { C } & \text { C22 } & -3.4165762 & -1.2346205 & 0.1071117 \\ 34 & \text { C } & \text { C23 } & -4.8146346 & -3.6694464 & -0.1960329 \\ 35 & \text { C } & \text { C24 } & -4.8236065 & -1.2362354 & -0.1934915 \\ 36 & \text { C } & \text { C25 } & -2.7298126 & 0.0002800 & 0.2578881 \\ 37 & \text { H } & \text { H7 } & -5.3406941 & -4.6130800 & -0.3098765 \\ 38 & \text { H } & \text { H11 } & -6.5560487 & -2.4875376 & -0.5619421 \\ 39 & \text { C } & \text { C26 } & -3.4168012 & 1.2348394 & 0.1052085 \\ 40 & \text { C } & \text { C27 } & -2.7481292 & 2.4880220 & 0.2414556 \\ 41 & \text { C } & \text { C28 } & -4.8238541 & 1.2357808 & -0.1954489 \\ 42 & \text { H } & \text { H15 } & -6.5565144 & 2.4861140 & -0.5659888 \\ 43 & \text { C } & \text { C29 } & -5.4992007 & -0.0003856 & -0.3370148 \\ 44 & \text { C } & \text { C30 } & -3.4246976 & 3.6701872 & 0.0920802 \\ 45 & \text { H } & \text { H16 } & -1.6867321 & 2.4825437 & 0.4653603 \\ 46 & \text { H } & \text { H17 } & -2.8983499 & 4.6144346 & 0.1987107 \\ 47 & \text { C } & \text { C31 } & -4.8152842 & 3.6689235 & -0.2018695 \\ 48 & \text { H } & \text { H18 } & -5.3414681 & 4.6123048 & -0.3172392 \\ 49 & \text { C } & \text { C32 } & -5.4946407 & 2.4869894 & -0.3412870 \\ 50 & \text { C } & \text { C33 } & -6.8974135 & -0.0008858 & -0.6355675 \\ 51 & \text { N } & \text { N1 } & -8.0366829 & -0.0020160 & -0.8796831\end{array}$

Point Group $=\mathrm{C} 1$ Order $=1$ Nsymop $=1$ 


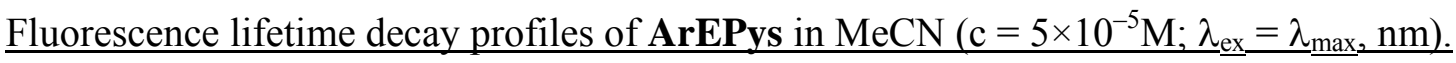
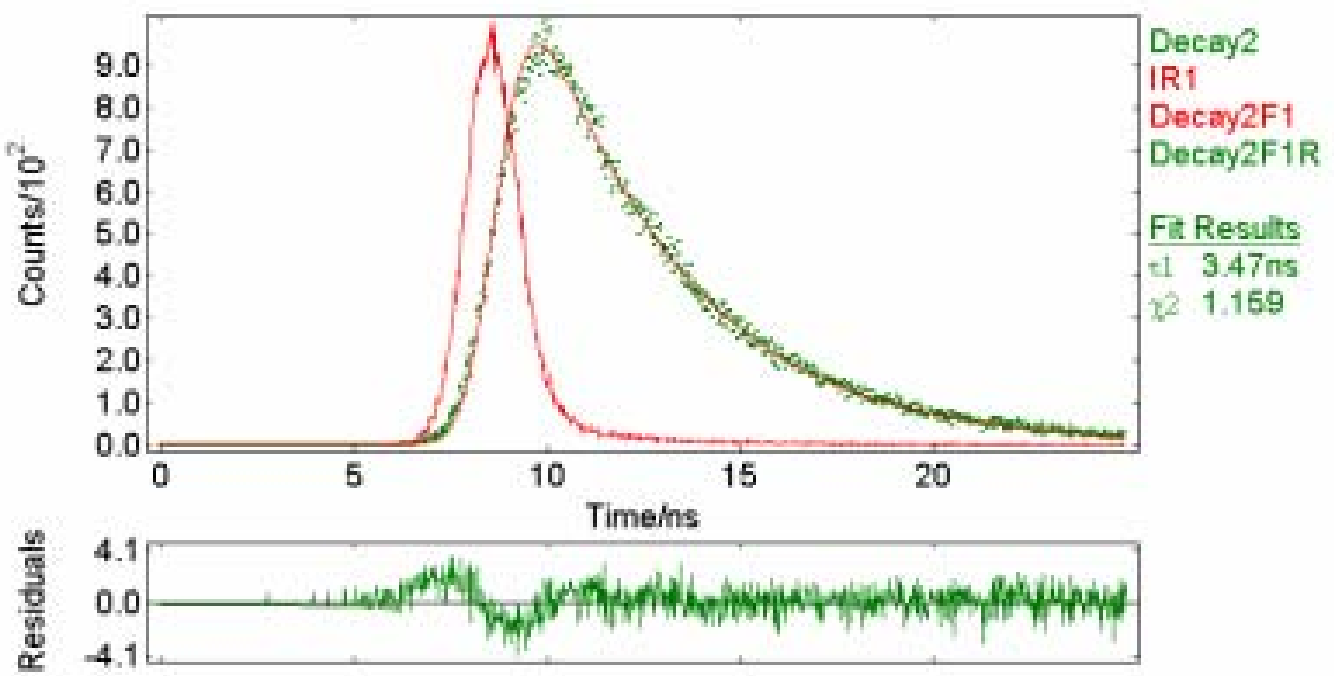

Fluorescence lifetime decay data for DMAEPy.
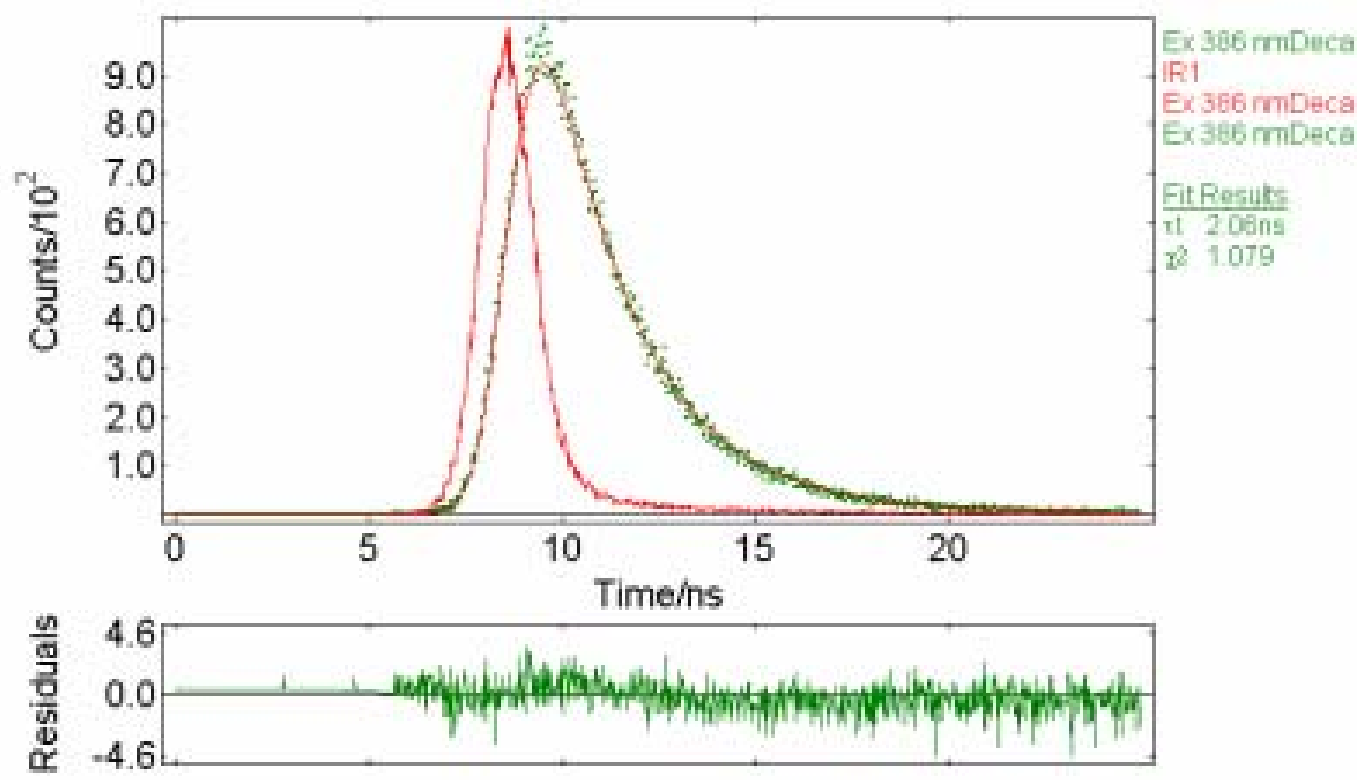

Fluorescence decay data for IPPEPy 


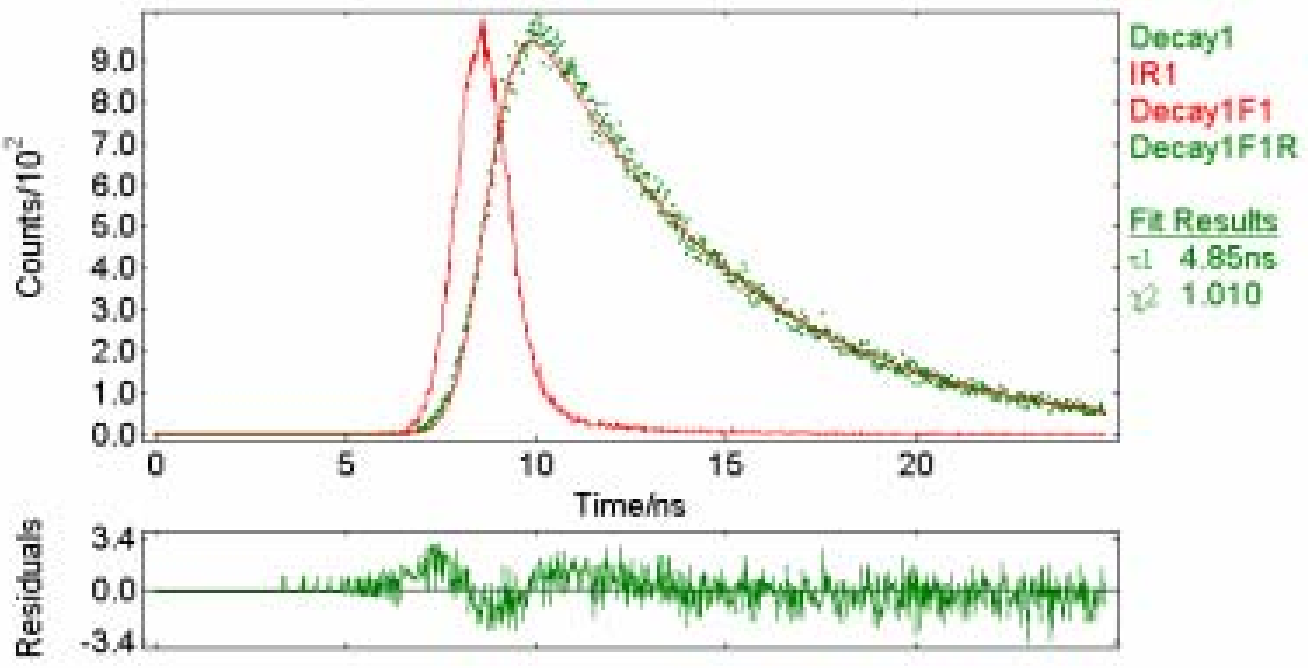

Decay date for PhEPy.

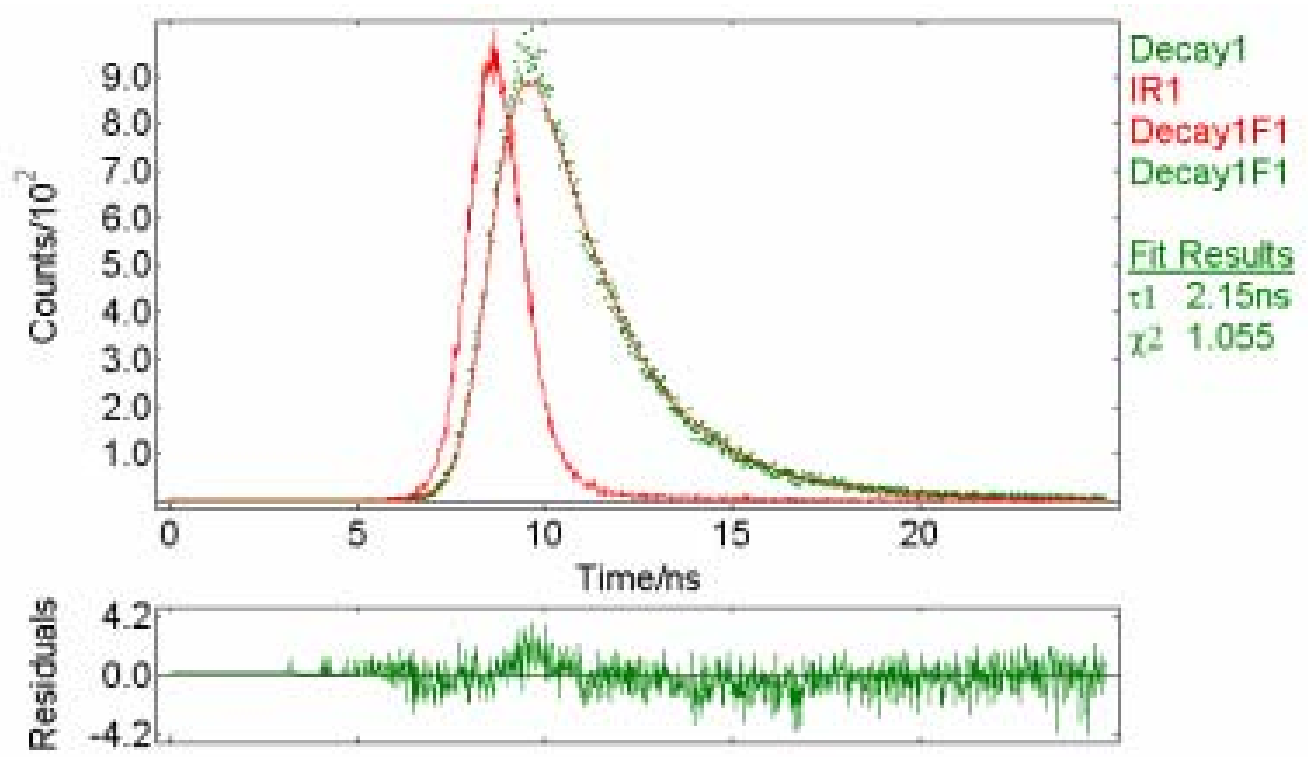

Fluorescence decay data for 2QEPy. 

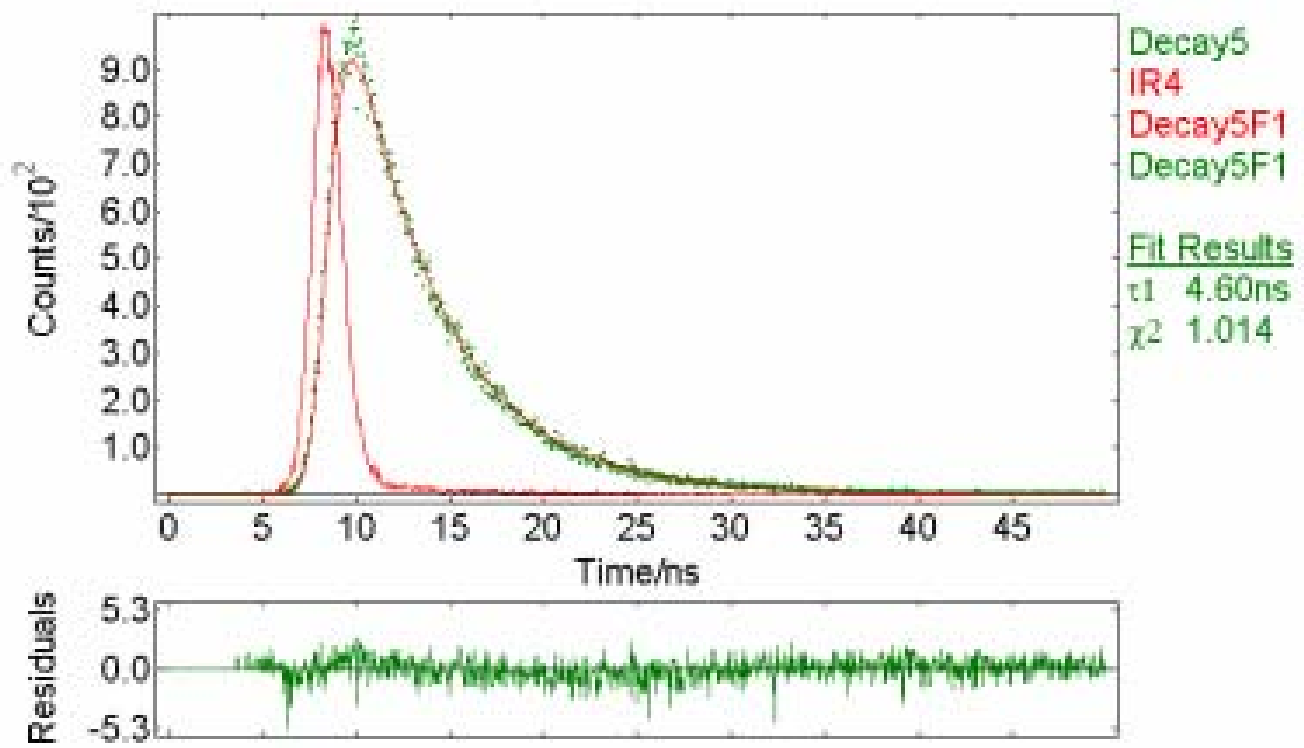

Decay data for ANEPy. 